\title{
On the structure and origin of pressure fluctuations in wall turbulence: predictions based on the resolvent analysis
}

\author{
M. Luhar ${ }^{1} \uparrow$, A. S. Sharma ${ }^{2}$ and B. J. McKeon ${ }^{1}$ \\ ${ }^{1}$ Graduate Aerospace Laboratories, California Institute of Technology, Pasadena, CA 91125, USA \\ ${ }^{2}$ Engineering and the Environment, University of Southampton, Southampton SO17 1BJ, UK
}

(Received 19 December 2013; revised 28 March 2014; accepted 16 May 2014; first published online 16 June 2014)

We generate predictions for the fluctuating pressure field in turbulent pipe flow by reformulating the resolvent analysis of McKeon and Sharma (J. Fluid Mech., vol. 658, 2010, pp. 336-382) in terms of the so-called primitive variables. Under this analysis, the nonlinear convective terms in the Fourier-transformed Navier-Stokes equations (NSE) are treated as a forcing that is mapped to a velocity and pressure response by the resolvent of the linearized Navier-Stokes operator. At each wavenumber-frequency combination, the turbulent velocity and pressure field are represented by the most-amplified (rank-1) response modes, identified via a singular value decomposition of the resolvent. We show that these rank-1 response modes reconcile many of the key relationships among the velocity field, coherent structure (i.e.hairpin vortices), and the high-amplitude wall-pressure events observed in previous experiments and direct numerical simulations (DNS). A Green's function representation shows that the pressure fields obtained under this analysis correspond primarily to the fast pressure contribution arising from the linear interaction between the mean shear and the turbulent wall-normal velocity. Recovering the slow pressure requires an explicit treatment of the nonlinear interactions between the Fourier response modes. By considering the velocity and pressure fields associated with the triadically consistent mode combination studied by Sharma and McKeon (J. Fluid Mech., vol. 728, 2013, pp. 196-238), we identify the possibility of an apparent amplitude modulation effect in the pressure field, similar to that observed for the streamwise velocity field. However, unlike the streamwise velocity, for which the large scales of the flow are in phase with the envelope of the small-scale activity close to the wall, we expect there to be a $\pi / 2$ phase difference between the large-scale wall-pressure and the envelope of the small-scale activity. Finally, we generate spectral predictions based on a rank-1 model assuming broadband forcing across all wavenumber-frequency combinations. Despite the significant simplifying assumptions, this approach reproduces trends observed in previous DNS for the wavenumber spectra of velocity and pressure, and for the scale-dependence of wall-pressure propagation speed.

Key words: boundary layer structure, boundary layers, pipe flow boundary layer

$†$ Email address for correspondence: mluhar@cantab.net 


\section{Introduction}

From noise generation to structural vibration and fatigue, the fluctuating pressure field induced by wall-bounded turbulent flows is important across a range of engineering problems. Further, the wall-pressure field is coupled to velocity fluctuations across the entire domain via a Poisson equation, and the wall-parallel gradient in wall pressure is directly proportional to the flux of vorticity from the wall (Koumoutsakos 1999). As a result, an improved understanding of the wall-pressure field can also contribute significantly to the development and implementation of effective control, especially because wall-based sensing represents the only truly practical option in most flows of engineering interest.

Unfortunately, there are many technical challenges associated with obtaining accurate wall-pressure measurements. The small spatial scales associated with turbulent flows impose severe sensor size limitations (Schewe 1983; Klewicki, Priyadarshana \& Metzger 2008). The presence of significant background noise and structural vibration results in inherently noisy measurements that require careful correction (Tsuji et al. 2007, 2012). Given these difficulties, our understanding of the wall-pressure field beneath turbulent flows lags behind our understanding of the fluctuating velocity fields.

Recent advances in direct numerical simulation (DNS) (e.g. Jimenez \& Hoyas 2008), along with improved pressure measurement techniques and the development of large-scale, high-Reynolds-number flow facilities (Tsuji et al. 2007; Klewicki et al. 2008) have provided significant insight into the statistical nature of the pressure field. However, the structural nature and origin of the pressure field is less well established. An accurate structural description requires temporally and spatially resolved pressure data. A characterization of the origin of these pressure structures also requires simultaneous velocity information. The resulting storage and processing requirements compound any technical challenges associated with obtaining such data in the first place.

\subsection{Structure and origin of wall-pressure fluctuations}

Broadly, numerical (Kim 1989; Jimenez \& Hoyas 2008) and experimental (Klewicki et al. 2008) results agree that, unlike the streamwise velocity fluctuations, wallpressure fluctuations tend to be circular in terms of aspect ratio (i.e.comparable streamwise and spanwise length scales). Laboratory measurements also suggest that the wall-pressure field consists of at least two distinct groups of structures. The first group consists of large-scale, low-frequency fluctuations that originate from velocity structures in the outer regions of the flow (e.g. Thomas \& Bull 1983; Snarski \& Lueptow 1995). The second group consists of high-frequency, small-scale disturbances that originate from structures in the buffer region of the flow (e.g. Schewe 1983; Johansson, Her \& Haritonidis 1987). These small-scale disturbances have been shown to be responsible for large-amplitude pressure spikes that contribute significantly to the long-time root-mean-square (r.m.s.) wall pressure. Further, recent measurements by Ghaemi \& Scarano (2013) show that these high-amplitude pressure peaks are correlated with distinct hairpin-like structures in the flow field.

DNS results (Kim 1989) and experimental observations (Schewe 1983; Johansson et al. 1987) suggest that the small-scale structures tend to have length scales $l^{+} \sim O(100-200)$ and propagate at speeds $c^{+} \approx 11-13$ (note: throughout this paper, a superscript + denotes normalization with respect to the inner units: friction velocity, $u_{\tau}$, and viscosity, v). Ghaemi \& Scarano (2013) suggest slightly higher propagation 
speeds for the high-amplitude pressure peaks, $c^{+} \approx 14$. Beyond this, there is no general consensus on the typical length scales and propagation speeds associated with the wall-pressure field. Broadband propagation speeds for wall pressure reported in the literature range from $0.5 U_{C L}$ to $0.8 U_{C L}$, where $U_{C L}$ represents the centreline or free-stream velocity depending on geometry (Bull 1967; Thomas \& Bull 1983; Kim 1989; Choi \& Moin 1990; Snarski \& Lueptow 1995; Hu, Morfey \& Sandham 2002). However, it is clear that a single broadband propagation speed is inappropriate for translating Eulerian wall-pressure measurements into structural features. Both DNS (e.g. Choi \& Moin 1990; Hu et al. 2002) and experiment (e.g. Bull 1967; Dinkelacker et al. 1977; Snarski \& Lueptow 1995; Klewicki et al. 2008) suggest a scale dependence; larger wall-pressure structures tend to propagate faster than smaller wall-pressure structures.

The spectral model developed by Panton \& Linebarger (1974) suggests that the propagation speed for wall pressure exhibits an overlap layer dominated by structures that scale linearly with distance from the wall, consistent with the attached-eddy hypothesis first proposed by Townsend and later developed by Perry and coworkers (e.g. Perry \& Chong 1982; Perry, Henbest \& Chong 1986; Perry \& Marusic 1995). More recently, Ahn, Graham \& Rizzi (2010) have extended the attached-eddy concept to generate a structure-based model for wall-pressure fluctuations. This model superposes contributions from individual eddies of an assumed horseshoe-like shape, with a number density that yields total circulation consonant with the mean velocity profile. The pressure contributions from individual eddies are estimated by solving the governing Poisson equation, assuming that the so-called fast source term arising from the linear interaction between the mean shear and the wall-normal velocity is dominant. Despite the simplifying assumptions, the model developed by Ahn et al. (2010) generates predictions which agree well with previous experiment and simulation, suggesting that the attached-eddy concept may also provide insight into the structure of the wall-pressure field.

The measurements of Thomas \& Bull (1983) suggest an interdependence between the large- and small-scale wall-pressure structures. Specifically, Thomas \& Bull (1983) observed that the smoothed, rectified high-frequency pressure fluctuations (i.e.the envelope of the high-frequency fluctuations) and the low-frequency fluctuations were correlated, and that the envelope of the high-frequency pressure fluctuations was approximately $\pi / 2$ out of phase with the low-frequency pressure fluctuations. In contrast, the envelope of the high-frequency wall shear stress was observed to be nearly in phase with the low-frequency wall shear stress. The latter result is consistent with the apparent amplitude modulation of the near-wall, small-scale velocity fluctuations due to the footprint of the so-called very large-scale motions (VLSMs) observed in previous studies (Bandhyopadhyay \& Hussain 1984; Mathis, Hutchins \& Marusic 2009; Marusic, Mathis \& Hutchins 2010; Mathis, Hutchins \& Marusic 2011). Hence, it may be possible to observe an apparent amplitude modulation in the wall-pressure field as well, albeit with a different phase relationship between the small and large scales. However, bear in mind that a direct comparison between the measurements of Thomas \& Bull (1983) and the more recent amplitude modulation studies is not strictly appropriate. The experiments of Thomas \& Bull (1983) were performed at lower Reynolds number and the large-scale structures they observed had much smaller length scales, $\approx 0.5 \delta$ where $\delta$ is the boundary layer thickness, compared to the VLSMs of length $O(10 \delta-20 \delta)$ thought to modulate the near-wall velocity and shear stress (Marusic et al. 2010). 


\subsection{Outline}

To provide further insight into the structure and origin of the wall-pressure field under turbulent flows, this paper extends the resolvent analysis proposed by McKeon \& Sharma (2010). For brevity, we only consider turbulent pipe flow. However, given the broad similarity in flow characteristics across the canonical flow configurations (pipes, channels, boundary layers; Monty et al. 2009), we expect our results to be generally applicable, at least in the near-wall region.

The resolvent analysis interprets the Navier-Stokes equations (NSE), Fourier transformed in the streamwise and azimuthal directions, and in time, as a forcingresponse system. The nonlinear convective terms in the NSE are treated as the forcing which creates the turbulent velocity field in response. The transfer function that maps this forcing to a velocity response is the well-known resolvent operator. Note that Fourier modes are the most appropriate bases for decomposition in the homogeneous directions. To identify an appropriate basis for the inhomogeneous wall-normal direction, the resolvent analysis employs a gain-based decomposition. Specifically, at each wavenumber-frequency combination, a singular value decomposition of the resolvent operator identifies the forcing and response modes (i.e.profiles in the wall-normal direction) that have the highest input-output gain. In other words, this decomposition identifies the velocity response that is most amplified for an unstructured forcing to the system. McKeon \& Sharma (2010) show that, in general, these highly amplified response modes are helical, propagating velocity structures that resemble three-dimensional versions of the near-singular critical-layer solutions obtained via classical Orr-Sommerfeld-Squire analyses.

More recently, Sharma \& McKeon (2013) have shown that structures resembling hairpin vortices arise naturally from the superposition of a pair of obliquely propagating velocity response modes, with azimuthal wavenumber $\pm n$. More complex structures, such as modulating packets of hairpin vortices, arise from the superposition of three mode pairs that are triadically consistent in terms of frequency and wavenumber. Sharma \& McKeon (2013) consider such mode combinations to be turbulence kernels. In this paper, we describe the wall-pressure field associated with such model structures. In particular, we consider the relationship between the velocity and pressure fields associated with the wavenumber-frequency combinations shown in table 1 at Reynolds number $R e=2 \bar{U} R / v=75000\left(R^{+}=1800\right)$, where $\bar{U}$ is the bulk-averaged mean velocity and $R$ is pipe radius. This Reynolds number corresponds roughly to the experimental conditions of Thomas \& Bull (1983) and Johansson et al. (1987), and the highest Reynolds number achieved in DNS by Jimenez \& Hoyas (2008). The smaller, near-wall mode $\boldsymbol{k}_{b}$ serves as a model for the high-intensity high-frequency buffer layer structures observed in previous experiments. The combination of triadically consistent modes $\boldsymbol{k}_{1}, \boldsymbol{k}_{2}$, and $\boldsymbol{k}_{3}$ (table 1) represents the turbulence kernel studied by Sharma \& McKeon (2013). Here, we extend this kernel to include pressure information, and we employ it to consider the relationship between the small- and large-scale wall-pressure field.

The remainder of this paper is structured as follows: $\S 2$ describes the extension of the resolvent analysis of McKeon \& Sharma (2010) to yield pressure information. We also develop a Green's function solution to provide further insight into the origin of the pressure field. Section 3 describes the velocity and pressure fields predicted for individual wavenumber-frequency combinations (particularly mode $\boldsymbol{k}_{b}$ ), and compares the predictions to experimental observations. Section 4 presents results pertaining to the turbulence kernel comprising modes $\boldsymbol{k}_{1}, \boldsymbol{k}_{2}$, and $\boldsymbol{k}_{3}$. In $\S 5$, we make additional simplifying assumptions to yield predictions for wavenumber spectra (§5.1), and propagation speed (\$5.2). Conclusions are presented in $\S 6$. 


\begin{tabular}{cccc}
\multicolumn{1}{c}{$k\left(\lambda_{x}^{+}\right)$} & $n\left(\lambda_{\theta}^{+}\right)$ & $c=\omega / k\left(c^{+}\right)$ & $a$ \\
Near-wall mode & & & \\
$\boldsymbol{k}_{b} \pm 60(190)$ & $\pm 60(190)$ & $1 / 2(12.5)$ & \\
Turbulence kernel & & & \\
$\boldsymbol{k}_{1} \pm 6(1900)$ & $\pm 6(1900)$ & $2 / 3(16.6)$ & 1 \\
$\boldsymbol{k}_{2} \pm 1(11500)$ & $\pm 6(1900)$ & $2 / 3(16.6)$ & 4.5 \\
$\boldsymbol{k}_{3} \pm 7(1650)$ & $\pm 12(960)$ & $2 / 3(16.6)$ & $-0.83 \mathrm{i}$
\end{tabular}

TABLE 1. Streamwise $(k)$ and spanwise $(n)$ wavenumbers, and speed $c$ for modes used frequently in this paper. The wavenumbers are normalized by pipe radius, and the speed is normalized by the mean centreline velocity. Also shown are inner-normalized estimates for the wavelength $\left(\lambda_{x}^{+}, \lambda_{\theta}^{+}\right)$and the wave speed $\left(c^{+}\right)$at Reynolds number $R e=75000\left(R^{+}=\right.$ 1800). The amplitude, $a$, describes the relative magnitude and phase of the three triadically consistent modes $\boldsymbol{k}_{1}, \boldsymbol{k}_{2}$, and $\boldsymbol{k}_{3}$ that make up the turbulence kernel considered in $\S 4$.

\section{Approach}

A summary of previous studies within the broad resolvent analysis framework can be found in McKeon, Jacobi \& Sharma (2013). In this paper, we develop the resolvent analysis further to better understand the pressure field in wall-bounded turbulent flows.

\subsection{Resolvent analysis}

We consider fully developed turbulent pipe flow. In the light of the cylindrical geometry, the statistical homogeneity in the streamwise direction, and stationarity in time, the turbulent velocity $(\tilde{\boldsymbol{u}})$ and pressure $(\tilde{p})$ fields can be expressed as a superposition of Fourier modes with streamwise wavenumber $k$, azimuthal wavenumber $n$ (constrained to be an integer), and frequency $\omega$ :

$$
\left[\begin{array}{l}
\tilde{\boldsymbol{u}}(x, y, \theta, t) \\
\tilde{p}(x, y, \theta, t)
\end{array}\right]=\sum_{n} \int_{-\infty}^{\infty} \int_{-\infty}^{\infty}\left[\begin{array}{l}
\boldsymbol{u}_{\boldsymbol{k}}(y) \\
p_{\boldsymbol{k}}(y)
\end{array}\right] \mathrm{e}^{\mathrm{i}(k x+n \theta-\omega t)} \mathrm{d} k \mathrm{~d} \omega
$$

where $x$ and $\theta$ are the streamwise and azimuthal coordinates, and $t$ is time. The wallnormal coordinate is $y=1-r$, where $r$ is the radial coordinate normalized by the pipe radius, $R$. With this decomposition, each wavenumber-frequency combination $\boldsymbol{k}=$ $(k, n, c=\omega / k)$ represents a helical wave propagating downstream with speed $c$.

The mean velocity profile and pressure field are expressed as $\boldsymbol{u}_{0}=(U(y), 0,0)$ and $p_{0}=P(x)$, and the fluctuating velocity and pressure fields are expressed as $\boldsymbol{u}=$ $(u, v, w)=\tilde{\boldsymbol{u}}-\boldsymbol{u}_{0}$ and $p=\tilde{p}-p_{0}$. The first $(U, u)$, second $(v)$, and third $(w)$ components of the velocity field represent the streamwise, wall-normal, and azimuthal velocities, respectively. Note that this paper employs both radial and wall-normal coordinates (figure 1). For notational convenience, most mathematical operations are presented in terms of the radial coordinate $(r)$ and velocity $(\hat{v})$. To ensure consistency with boundary layer and channel flow studies, the results are presented almost exclusively in terms of the wall-normal coordinate $(y)$ and velocity $(v)$.

Under the Fourier representation shown in (2.1), at each wavenumber-frequency combination $\boldsymbol{k}$ the NSE for turbulent pipe flow can be expressed in dimensionless form as:

$$
\begin{gathered}
(-\mathrm{i} \omega+\mathrm{i} k U) \boldsymbol{u}_{k}+v_{k} U^{\prime} \boldsymbol{e}_{x}+\nabla p_{k}-R e^{-1} \nabla^{2} \boldsymbol{u}_{k}=\boldsymbol{f}_{k}, \\
\nabla \cdot \boldsymbol{u}_{k}=0 .
\end{gathered}
$$




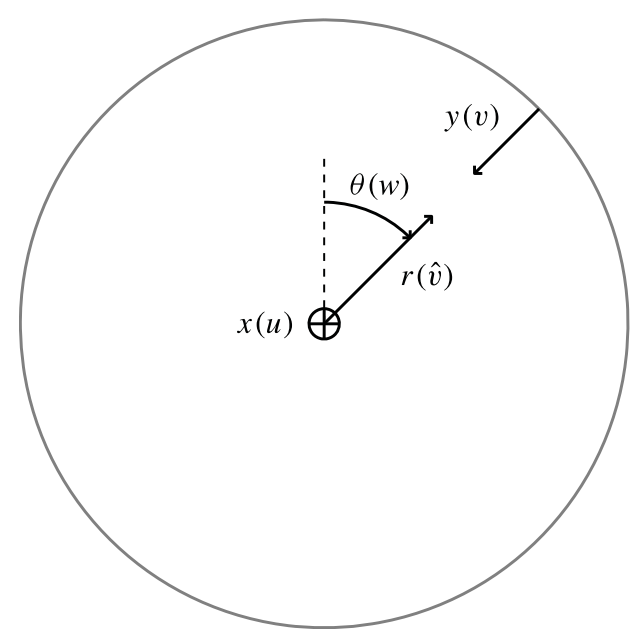

FIGURE 1. Schematic showing coordinate system. The $x$-axis goes into the page.

Here, $R e$ is the Reynolds number, $U^{\prime}$ is the mean shear $\boldsymbol{e}_{\boldsymbol{x}}$ is the unit vector in the streamwise direction, and the nonlinear convective terms at each wavenumberfrequency combination are lumped into a forcing term, $\boldsymbol{f}_{\boldsymbol{k}}=(-\boldsymbol{u} \cdot \nabla \boldsymbol{u})_{\boldsymbol{k}}$. Equations (2.2) and (2.3) can be re-arranged to arrive at the following input-output (or forcing-response) relationship:

$$
\begin{aligned}
{\left[\begin{array}{l}
\boldsymbol{u}_{k} \\
p_{k}
\end{array}\right] } & =\left(-\mathrm{i} \omega\left[\begin{array}{ll}
\boldsymbol{I} & \\
& 0
\end{array}\right]-\left[\begin{array}{cc}
\mathscr{L}_{k} & -\nabla \\
\nabla^{\mathrm{T}} & 0
\end{array}\right]\right)^{-1}\left[\begin{array}{l}
\boldsymbol{I} \\
0
\end{array}\right] \boldsymbol{f}_{\boldsymbol{k}} \\
& =\tilde{\mathscr{H}}_{\boldsymbol{k}} \boldsymbol{f}_{\boldsymbol{k}} .
\end{aligned}
$$

The nonlinear terms $\left(f_{k}\right)$ are interpreted as a forcing to the linear Navier-Stokes system, and the resolvent operator, $\tilde{\mathscr{H}}_{\boldsymbol{k}}$, maps this forcing to velocity $\left(\boldsymbol{u}_{\boldsymbol{k}}\right)$ and pressure $\left(p_{k}\right)$ responses. In (2.4), $\boldsymbol{I}$ is the identity operator, $\nabla$ and $\nabla^{\mathrm{T}}$ represent the gradient and divergence operators, and $\mathscr{L}_{k}(\boldsymbol{k}, U, R e)$ is the linear Navier-Stokes operator:

$$
\mathscr{L}_{k}=\left[\begin{array}{ccc}
-\mathrm{i} k U+\frac{\mathrm{D}+r^{-2}}{R e} & -\frac{\partial U}{\partial r} & 0 \\
0 & -\mathrm{i} k U+\frac{\mathrm{D}}{R e} & -\frac{2 \mathrm{i} n r^{-2}}{R e} \\
0 & \frac{2 \mathrm{i} n r^{-2}}{R e} & -\mathrm{i} k U+\frac{\mathrm{D}}{R e}
\end{array}\right]
$$

where $\mathrm{D}=-k^{2}-\left(n^{2}+1\right) r^{-2}+\partial_{r}^{2}+r^{-1} \partial_{r}$ represents the Laplacian.

The original resolvent analysis of McKeon \& Sharma (2010) projected the NSE onto a series of divergence-free basis functions that satisfied the correct boundary conditions at the wall $\left(\boldsymbol{u}_{k}=0\right)$. This projection satisfies mass continuity and eliminates the pressure term in the momentum equations (Meseguer \& Trefethen 2003). Instead of employing this projection, in this paper we formulate the resolvent operator directly from the so-called primitive variable (2.4). Pressure and mass continuity are retained explicitly. This extension permits direct access to pressure information, and it also allows consideration of alternative boundary conditions (e.g. Luhar, Sharma \& McKeon 2013, 2014).

Following McKeon \& Sharma (2010), we perform a singular value decomposition of the resolvent operator $\tilde{\mathscr{H}}_{k}$, discretized using a Chebyshev pseudospectral method, 
to identify high-gain forcing and response directions under an energy norm. To avoid the singularity at $r=0$, we follow the method outlined in Trefethen (2000, Chapter 11). The grid is generated on an even number $(2 N)$ of points for $r \in[-1,1]$. The $N$ points for $r \in(0,1]$ are retained and the $[N \times N]$ matrices for differentiation are formulated from their $[2 N \times 2 N]$ counterparts by taking advantage of the appropriate odd/even symmetry conditions across $r=0$ for the velocity and pressure fields. To enforce an energy norm within the primitive-variable formulation employed here, the forcing-response system shown in (2.4) is scaled such that:

$$
\left[\begin{array}{ll}
W_{u} & 0
\end{array}\right]\left[\begin{array}{l}
\boldsymbol{u}_{k} \\
p_{k}
\end{array}\right]=\left(\left[\begin{array}{ll}
W_{u} & 0
\end{array}\right] \tilde{\mathscr{H}}_{\boldsymbol{k}} W_{f}^{-1}\right) W_{f} \boldsymbol{f}_{k}
$$

or

$$
W_{u} \boldsymbol{u}_{k}=\tilde{\mathscr{H}}_{\boldsymbol{k}}^{S} W_{f} \boldsymbol{f}_{\boldsymbol{k}}
$$

Here, the discretized resolvent operator $\tilde{\mathscr{H}}_{\boldsymbol{k}}$ is a $[4 \times 3]$ block matrix and the scaled resolvent operator $\tilde{\mathscr{H}}_{k}^{S}$ is a [3×3] block matrix. The [3 3 3] block diagonal matrices $W_{u}$ and $W_{f}$ incorporate numerical quadrature weights which ensure that a singular value decomposition of the scaled resolvent operator:

$$
\tilde{\mathscr{H}}_{k}^{S}=\sum_{m} \psi_{k, m} \sigma_{k, m} \phi_{k, m}^{*}
$$

where

$$
\begin{gathered}
\sigma_{k, 1}>\sigma_{k, 2} \cdots>\sigma_{k, m}>0, \\
\phi_{k, l}^{*} \phi_{k, m}=\delta_{l m}, \quad \psi_{k, l}^{*} \psi_{k, m}=\delta_{l m},
\end{gathered}
$$

yields forcing modes $\boldsymbol{f}_{\boldsymbol{k}, m}=W_{f}^{-1} \phi_{\boldsymbol{k}, m}$ and velocity response modes $\boldsymbol{u}_{\boldsymbol{k}, m}=W_{\boldsymbol{u}}^{-1} \psi_{\boldsymbol{k}, m}$ with unit energy over the pipe cross-section. In other words, with the scaling shown in (2.6) or (2.7) the orthonormality conditions shown in (2.10) translate to:

$$
\int_{0}^{1} \boldsymbol{f}_{\boldsymbol{k}, l}^{*} \boldsymbol{f}_{\boldsymbol{k}, m} r \mathrm{~d} r=\delta_{l m}, \quad \int_{0}^{1} \boldsymbol{u}_{\boldsymbol{k}, l}^{*} \boldsymbol{u}_{\boldsymbol{k}, m} r \mathrm{~d} r=\delta_{l m} .
$$

The superscript * in (2.10) and (2.11) denotes a conjugate transpose.

From (2.6) or (2.7), and (2.8), it is evident that if forcing is aligned in the direction of $\boldsymbol{f}_{\boldsymbol{k}, m}=W_{f}^{-1} \phi_{k, m}$ with unit energy, a velocity response is created in the direction of $\boldsymbol{u}_{k, m}=W_{\boldsymbol{u}}^{-1} \psi_{\boldsymbol{k}, m}$ with energy $\sigma_{\boldsymbol{k}, m}^{2}$. Thus, for unit forcing across all $\boldsymbol{f}_{\boldsymbol{k}, m}$, the most energetic (i.e. highest $\sigma_{k}$ ) velocity response occurs in the direction of $\boldsymbol{u}_{k, 1}$.

Due to the energy norm imposed through the scaling in (2.6) or (2.7), the singular value decomposition of the scaled resolvent operator (2.8) does not immediately yield the pressure fields, $p_{k, m}$, associated with the response modes, $\boldsymbol{u}_{k, m}$. The pressure field is recovered by reverting to the unscaled formulation shown in (2.4). Specifically, we have:

$$
\sigma_{k, m}\left[\begin{array}{l}
\boldsymbol{u}_{k, m} \\
p_{\boldsymbol{k}, m}
\end{array}\right]=\tilde{\mathscr{H}}_{\boldsymbol{k}} \boldsymbol{f}_{\boldsymbol{k}, m}
$$

Note that an energy norm is a natural choice for the gain analysis pursued here. Under this norm, the singular value decomposition identifies the forcing direction that leads to the most amplified, in terms of kinetic energy, velocity response. However, 
bear in mind that alternative norms can also be imposed by scaling the resolvent appropriately. For instance, the scaling matrix $W_{u}$ may be altered such that the integrals in (2.11) weight each component of velocity differently. Alternatively, the scaling factor $\left[\begin{array}{ll}W_{u} & 0\end{array}\right]$ in (2.6), which only weights the velocity components, may be replaced by something that only weights the pressure field, $\left[\begin{array}{ll}0 & W_{p}\end{array}\right]$, such that the singular value decomposition identifies the forcing modes that lead to the largest pressure response. We refer to this alternative scaling as a pressure norm.

Based on the form of the resolvent operator in (2.4), McKeon \& Sharma (2010) suggest that large amplification (high $\sigma_{k}$ ) can arise through two mechanisms: via the linear coupling between mean shear $\left(U^{\prime}\right)$ and wall-normal velocity $\left(v_{k}\right)$, which is responsible for the non-normal nature of the resolvent operator; and when the velocity responses are localized near a critical layer, $y_{c}$, where the phase speed of the modes matches the local mean velocity, $c=\omega / k=U\left(y_{c}\right)$, such that the diagonal term is $(k U-\omega) \approx 0$. In the latter case, the velocity response modes closely resemble the critical-layer solutions obtained from classical linear Orr-Sommerfeld-Squire analyses. Extending the concepts of linear stability analysis to the present turbulent case, the first singular response modes $\boldsymbol{u}_{k, 1}$ can loosely be interpreted as the least-damped velocity fields at the wavenumber-frequency combinations $\boldsymbol{k}$. They are sustained by minimal forcing in the direction of $f_{k, 1}$.

Importantly, a recent study by Moarref et al. (2013) shows that the resolvent operators are low-rank at wavenumber-frequency combinations that are energetic in real turbulence. Only a limited number of input directions are highly amplified (i.e. $\sigma_{k, m}$ decreases sharply with increasing $m$ ) and so the velocity field $\boldsymbol{u}_{\boldsymbol{k}}$ may be reasonably approximated by combining the first few singular response modes. In many cases, the first singular response mode, $\boldsymbol{u}_{k, 1}$, tends to be so highly amplified that it is expected to dominate the velocity field at that wavenumber-frequency combination, as long as a non-zero component of forcing exists along $f_{k, 1}$ in the real flow. Indeed, Moarref et al. (2013) and Sharma \& McKeon (2013) show that many of the key statistical and structural properties of wall turbulence can be captured with rank-1 models (i.e. $\boldsymbol{u}_{\boldsymbol{k}} \propto \boldsymbol{u}_{k, 1}$ ). Therefore, we consider only the first singular response modes for the remainder of this paper. For notational convenience, we drop the additional subscript 1.

Note that the resolvent analysis only predicts the velocity response mode shape $\left(\boldsymbol{u}_{k}\right)$ and gain $\left(\sigma_{k}\right)$ at each wavenumber-frequency combination. It does not predict the relative amplitude and phase of each $\boldsymbol{u}_{\boldsymbol{k}}$ present in the real flow. Throughout this paper, we use the term resolvent modes to refer to the normalized velocity fields $\boldsymbol{u}_{\boldsymbol{k}}$ obtained from the singular value decomposition (i.e. with unit energy across the pipe cross-section, (2.11)). As is evident from (2.4)-(2.10), the amplitude and phase of each resolvent mode $\boldsymbol{u}_{k}$ present in the flow depends on the amplitude and phase of the nonlinear forcing $f_{k}$.

Note also that construction of the resolvent operators (2.4) requires information regarding the mean velocity profile, $U$. For simplicity, in this paper we assume a mean velocity profile, obtained from experimental (McKeon et al. 2004) or DNS (Wu \& Moin 2008) data. In a more complete model, the mean velocity profile must be sustained through the summation of the mean Reynolds stress contributions from individual modes. Since a wide range of wavenumber-frequency combinations contribute to the mean Reynolds stress, this requires consideration of many resolvent modes and an explicit treatment of the link between $\boldsymbol{u}$ and $\boldsymbol{f}$ (McKeon et al. 2013). 


\subsection{Pressure Poisson equation and Green's function solution}

To better understand the origin of the pressure fields obtained under the resolvent analysis, we consider a Green's function representation. Taking the divergence of the momentum equation (2.2) yields the Poisson equation for the turbulent pressure field in incompressible flows. The source terms in the pressure Poisson equation consist of a linear contribution arising from the product of the mean shear $\left(U^{\prime}\right)$ and streamwise gradient of wall-normal velocity $\left(\mathrm{i} k v_{k}\right)$, and a nonlinear contribution from the interaction between the fluctuating velocity components $\left(\nabla \cdot f_{k}\right)$ :

$$
\nabla^{2} p_{k}=-2 \mathrm{i} k v_{k} U^{\prime}+\nabla \cdot f_{k} .
$$

From the wall-normal momentum equation, it can be shown that the fluctuating pressure field must satisfy the following inhomogeneous Neumann boundary condition at the wall:

$$
\left.\frac{\partial p_{k}}{\partial r}\right|_{r=1}=\frac{1}{R e}\left[\frac{1}{r} \frac{\partial}{\partial r}\left(r \frac{\partial \hat{v}_{k}}{\partial r}\right)\right]_{r=1} .
$$

Following previous literature (e.g. Kim 1989), we split the pressure field into three separate components: the so-called fast pressure $\left(p_{k, f}\right)$ generated due to the linear source terms, the slow pressure $\left(p_{k, s}\right)$ due to the nonlinear source terms, and the Stokes pressure $\left(p_{k, s t}\right)$ due to the inhomogeneous boundary condition. These pressure components individually satisfy the following equations:

$$
\begin{gathered}
\nabla^{2} p_{k, f}=-2 \mathrm{i} k v_{\boldsymbol{k}} U^{\prime},\left.\quad \frac{\partial p_{\boldsymbol{k}, f}}{\partial r}\right|_{r=1}=0, \\
\nabla^{2} p_{\boldsymbol{k}, s}=\nabla \cdot \boldsymbol{f}_{\boldsymbol{k}},\left.\quad \frac{\partial p_{\boldsymbol{k}, s}}{\partial r}\right|_{r=1}=0, \\
\nabla^{2} p_{k, s t}=0,\left.\quad \frac{\partial p_{\boldsymbol{k}, s t}}{\partial r}\right|_{r=1}=\frac{1}{R e}\left[\frac{1}{r} \frac{\partial}{\partial r}\left(r \frac{\partial \hat{v}_{\boldsymbol{k}}}{\partial r}\right)\right]_{r=1} .
\end{gathered}
$$

The DNS of Kim (1989) and Jimenez \& Hoyas (2008) suggest that the slow pressure tends to be larger than the fast pressure, except very close to the wall, where the fast pressure is more important. However, in general, the magnitude of both the slow and fast terms is comparable. The Stokes pressure tends to be negligible at high Reynolds number.

For a source at $r^{\prime}$, the Green's function for the fast and slow components of pressure in turbulent pipe flow satisfies:

$$
\begin{gathered}
\nabla^{2} G_{k}=\frac{1}{r} \frac{\partial}{\partial r}\left(r \frac{\partial G_{k}}{\partial r}\right)-\left(k^{2}+\frac{n^{2}}{r^{2}}\right) G_{k}=\frac{1}{r} \delta\left(r-r^{\prime}\right), \\
\left.\frac{\partial G_{k}}{\partial r}\right|_{r=1}=0
\end{gathered}
$$

where $\delta$ represents the Dirac delta. Integrating (2.18) across the delta function yields the following matching conditions at the source:

$$
\begin{gathered}
\left.\frac{\partial G_{k}}{\partial r}\right|_{r^{\prime}+\epsilon}-\left.\frac{\partial G_{k}}{\partial r}\right|_{r^{\prime}-\epsilon} \underset{\epsilon \rightarrow 0}{=} \frac{1}{r^{\prime}}, \\
\left.G_{k}\right|_{r^{\prime}+\epsilon}-\left.G_{k}\right|_{r^{\prime}-\epsilon}=0 .
\end{gathered}
$$


For $r^{\prime} \neq r,(2.18)$ represents a modified Bessel equation. So, the Green's function can be expressed in terms of the modified Bessel functions, $\mathrm{I}_{n}$ and $\mathrm{K}_{n}$. With some algebra, it can be shown that the solution to (2.18) that satisfies (2.19)-(2.21) for $k \neq 0$ and $n \neq 0$ is:

$$
G_{k}\left(r, r^{\prime}\right)= \begin{cases}-A_{k} \mathrm{I}_{n}\left(k r^{\prime}\right) \mathrm{I}_{n}(k r)-\mathrm{I}_{n}\left(k r^{\prime}\right) \mathrm{K}_{n}(k r), & 1 \geqslant r \geqslant r^{\prime}, \\ -A_{k} \mathrm{I}_{n}\left(k r^{\prime}\right) \mathrm{I}_{n}(k r)-\mathrm{I}_{n}(k r) \mathrm{K}_{n}\left(k r^{\prime}\right), & 0 \leqslant r \leqslant r^{\prime},\end{cases}
$$

with

$$
A_{k}=\frac{\mathrm{K}_{n-1}(k)+\mathrm{K}_{n+1}(k)}{\mathrm{I}_{n-1}(k)+\mathrm{I}_{n+1}(k)}
$$

(cf. the Green's function for turbulent channel flow, Kim 1989). Using $G_{k}$, the fast and slow pressure become:

$$
\begin{gathered}
p_{k, f}(r)=\int_{0}^{1} G_{k}\left(r, r^{\prime}\right)\left(-\left.2 \mathrm{i} k \hat{v}_{k} \frac{\partial U}{\partial r}\right|_{r^{\prime}}\right) r^{\prime} \mathrm{d} r^{\prime}, \\
p_{k, s}(r)=\int_{0}^{1} G_{k}\left(r, r^{\prime}\right)\left(\nabla \cdot f_{k}\right) r^{\prime} \mathrm{d} r^{\prime} .
\end{gathered}
$$

Further, it is straightforward to show that the Stokes pressure satisfies:

$$
p_{k, s t}(r)=\left[\frac{\mathrm{I}_{n}(k r)}{\mathrm{I}_{n-1}(k)+\mathrm{I}_{n+1}(k)}\right] \frac{2}{k \operatorname{Re}}\left[\frac{1}{r} \frac{\partial}{\partial r}\left(r \frac{\partial \hat{v}_{k}}{\partial r}\right)\right]_{r=1} .
$$

Note that (2.18)-(2.26) have been expressed in terms of the radial coordinate, $r$. However, unless otherwise stated, the discussion below is framed in terms of the wall-normal coordinate $y=(1-r)$ and velocity.

The magnitude of the Green's function at the wall $(y=0)$ corresponding to a source located at the wall $\left(y^{\prime}=0\right)$ is shown in figure $2(a)$. It is clear that the magnitude of the Green's function decreases with increasing streamwise and spanwise wavenumbers. Thus, for identical source strengths, larger structures (lower $k, n$ ) generate stronger pressure fields. However, bear in mind that the source terms for the pressure Poisson equation (2.13) depend on gradients in the velocity fields. These gradients are likely to be larger for the smaller-scale structures.

Figure 2(b) shows the variation in the Green's functions at the wall $(y=0)$ for varying source locations $\left(y^{\prime}\right)$, for the four different wavenumber combinations shown in table $1: \boldsymbol{k}_{1}=(k, n)=(6,6), \boldsymbol{k}_{2}=(1,6)$, and $\boldsymbol{k}_{3}=(7,12)$, along with $\boldsymbol{k}_{b}=(60,60)$. The streamwise wavenumber $k$ is normalized based on pipe radius, such that $k=1$ corresponds to a streamwise wavelength $2 \pi R$. The Green's functions corresponding to higher wavenumbers (e.g. $\boldsymbol{k}_{b}, \boldsymbol{k}_{3}$ ) decay very rapidly away from the wall compared to those for the lower wavenumbers (e.g. $\boldsymbol{k}_{2}$ ). Thus, larger-scale structures in the velocity field are likely to have a longer-range influence on wall pressure.

\section{Individual resolvent modes}

In this section, we describe the structure of the velocity and pressure fields predicted under the resolvent analysis for the modes $\boldsymbol{k}_{b}$ and $\boldsymbol{k}_{1}$ (§3.1). These results are discussed in the context of previous experimental measurements linking high-amplitude pressure peaks to buffer-region velocity structures (Johansson et al. 

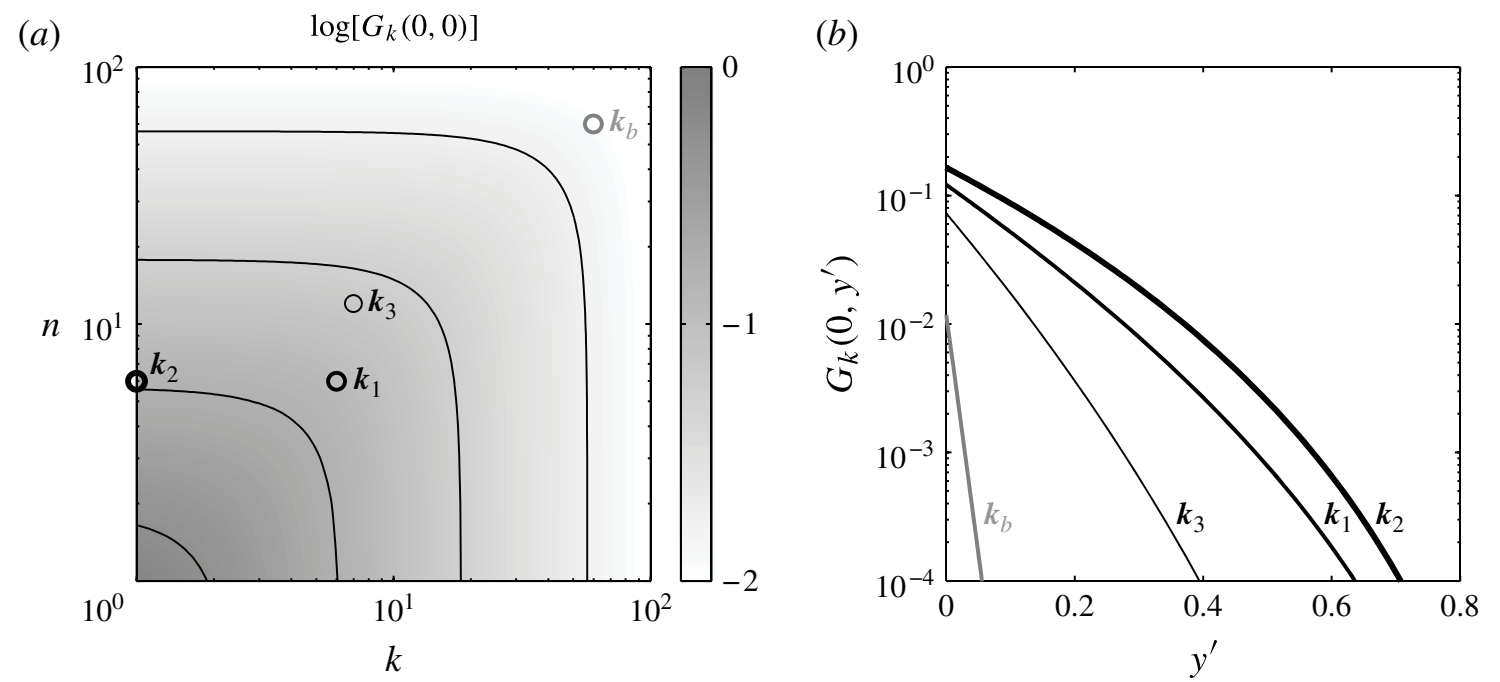

FIGURE 2. (a) Log-spaced contours showing the magnitude of the Green's function at the wall $(y=0)$ for source terms at the wall $\left(y^{\prime}=0\right)$. The solid lines show contours at $(-0.25,-0.75,-1.25,-1.75)$. (b) Green's functions for wall pressure for the four wavenumber combinations marked in $(a): \boldsymbol{k}_{1}=(6,6), \boldsymbol{k}_{2}=(1,6), \boldsymbol{k}_{3}=(7,12)$, and $\boldsymbol{k}_{b}=(60,60)$.

1987; Snarski \& Lueptow 1995), the recently observed link between the pressure peaks and structures resembling hairpin vortex packets (Ghaemi \& Scarano 2013), and the splat-spin interpretation of the pressure field proposed by Bradshaw \& Koh (1981) (\$3.2). We also consider the relative contributions of the fast, slow and Stokes pressure, and the effect of a pure pressure norm on the resolvent modes $(\S 3.3)$.

\subsection{Relationship between velocity and pressure fields}

Figure 3 shows the magnitude and phase of the velocity and pressure fields for the modes $\boldsymbol{k}_{b}$ and $\boldsymbol{k}_{1}$ at $R e=75000\left(R^{+}=1800\right)$. The mode $\boldsymbol{k}_{b}$ represents a helical wave of streamwise and spanwise wavelength $\lambda_{\theta}^{+} \approx \lambda_{x}^{+} \approx 190$, propagating downstream at $50 \%$ of the pipe centreline speed, $c^{+}=12.5$. The mode $\boldsymbol{k}_{1}$ represents a velocity structure of streamwise and spanwise wavelength $\lambda_{\theta}^{+} \approx \lambda_{x}^{+} \approx 1900$, propagating downstream at $2 / 3$ of the pipe centreline speed, $c^{+}=16.6$ (table 1 ). The critical layers for $\boldsymbol{k}_{b}$ and $\boldsymbol{k}_{1}$, where mode speed matches the local mean velocity $U^{+}\left(y_{c}^{+}\right)=c^{+}$, fall at $y_{c}^{+} \approx 23$ and $y_{c}^{+} \approx 110$, respectively. Although we only consider two wavenumber-frequency combinations in this section, the results presented below are generally representative of the velocity and pressure fields predicted under the resolvent analysis.

The velocity magnitude and phase profiles shown in figure 3 reveal some features common to many resolvent modes (McKeon \& Sharma 2010; McKeon et al. 2013; Sharma \& McKeon 2013). For instance, the magnitude of the streamwise velocity $u_{k}$ peaks at, or very near, the critical layer, while the wall-normal velocity $v_{k}$ peaks at a location slightly further away from the wall (figure $3 a, c$ ). Note that the velocity magnitudes are generally larger for mode $\boldsymbol{k}_{b}$ compared to mode $\boldsymbol{k}_{1}$ due to the energynormalization shown in (2.11). The resolvent modes have unit kinetic energy when integrated over the pipe cross-section. Since the smaller mode $\boldsymbol{k}_{b}$ has a smaller wallnormal extent, the magnitudes of the velocity fields are larger. 
(a)

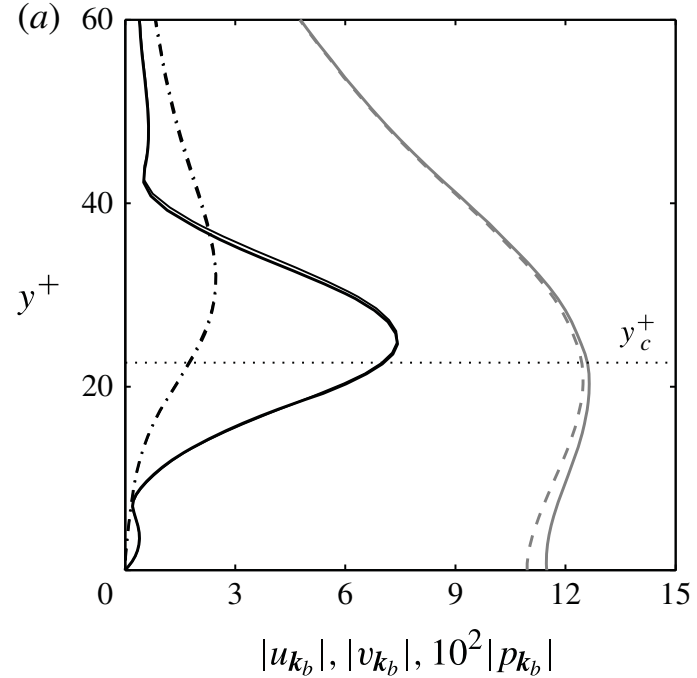

(c)

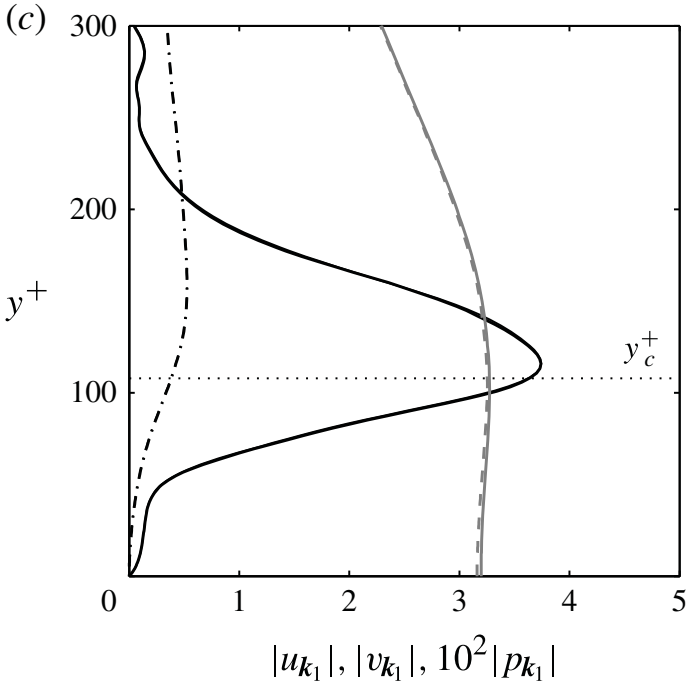

(b)

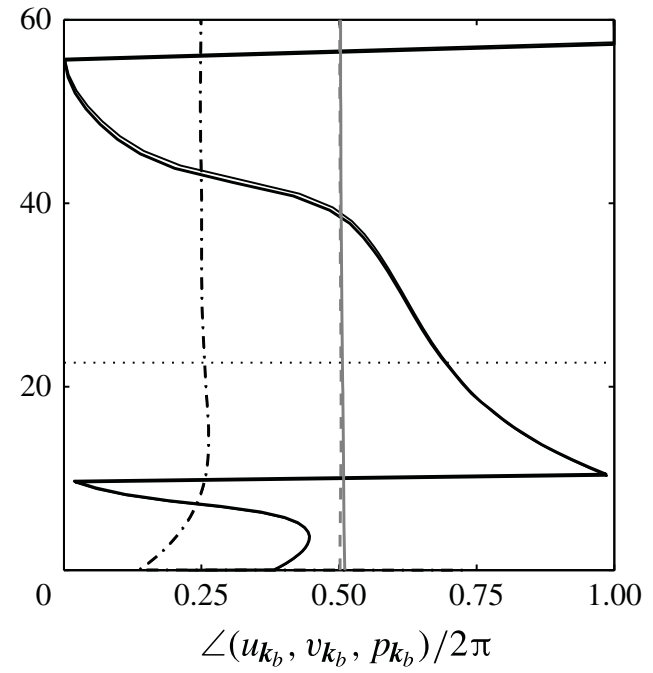

(d)

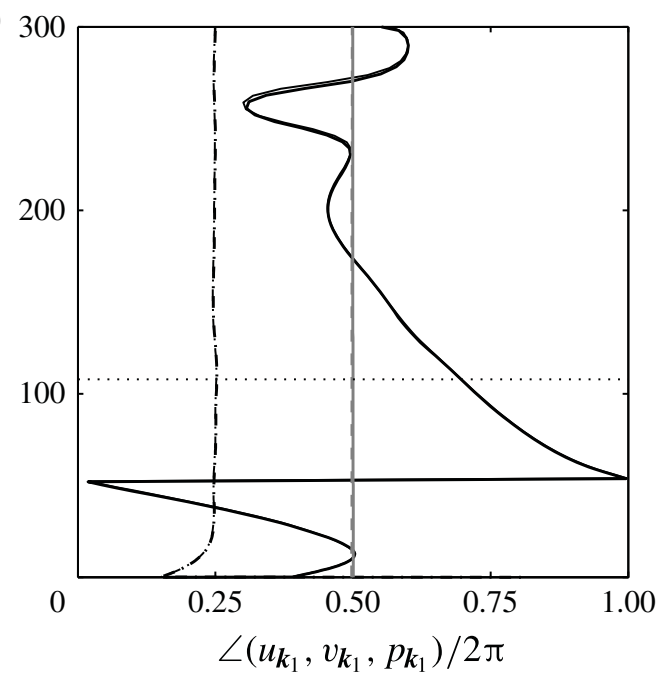

FigurE 3. Vertical profiles showing the magnitude $(a, c)$ and phase $(b, d)$ of the streamwise (bold black line) and wall-normal (dash-dotted black line) velocity, and the pressure field (solid grey line) for modes $\boldsymbol{k}_{b}(a, b)$ and $\boldsymbol{k}_{1}(c, d)$. The dashed grey lines show the pressure field predicted by a Green's function solution, assuming contributions only from the fast source term. The fine black lines represent the velocity fields predicted under the original resolvent analysis of McKeon \& Sharma (2010). Note that the bold and fine black lines are hard to distinguish because they are almost identical.

For both resolvent modes, the phase of the wall-normal velocity is approximately constant in $y$ (figure $3 b, d$ ), while the phase of the streamwise velocity decreases by $\pi$ across the critical layer, i.e. across $y_{c}^{+} \approx 10-40$ for mode $\boldsymbol{k}_{b}$ and across $y_{c}^{+} \approx 50-170$ for mode $\boldsymbol{k}_{1}$. Further, the wall-normal velocity is approximately $\pi$ out of phase with the streamwise velocity at the critical layer. These phase profiles are typical of the critical layer solutions expected from linear analysis of the Orr-Sommerfeld-Squire equations (McKeon \& Sharma 2010; McKeon et al. 2013). The $\pi$ phase difference between the wall-normal and streamwise velocity at the critical layer comes about due to the leading-order balance between the mean shear and viscous terms in the $u$-momentum equation. Specifically, $(-\mathrm{i} \omega+\mathrm{i} k U)=0$ at the critical layer and the 
nonlinear forcing term does not contribute significantly to the momentum balance for highly amplified response modes. As a result, we expect the following balance: $v_{k} U^{\prime} \sim$ $\nabla^{2} u_{k}$ (2.2). Since the mean shear is positive $\left(U^{\prime}>0\right)$, we have $\operatorname{sign}\left(v_{k}\right)=\operatorname{sign}\left(\nabla^{2} u_{k}\right)$ at the critical layer, i.e. the streamwise velocity is maximum (minimum) in regions of negative (positive) wall-normal velocity.

Note that figure 3 shows the velocity structure predicted by both the present primitive-variable resolvent analysis (bold black lines) and the projection-based analysis (fine black lines) pursued by McKeon \& Sharma (2010) at identical grid resolutions: $N=400$ points in $r \in(0,1]$. For the modes shown in table 1 , the singular values predicted by the present analysis converged to within $1 \%$ of those predicted by McKeon \& Sharma (2010) for $N>150$. Therefore, all the results presented in this paper employ grid resolutions $N \geqslant 150$. There are very minor differences in the magnitude and phase of the streamwise velocity near $y^{+} \approx 30$ for mode $\boldsymbol{k}_{b}$ (figure $3 a, b$ ). For mode $\boldsymbol{k}_{1}$ (figure $3 c, d$ ), the magnitude and phase of the velocity fields agree within plotting accuracy. For both modes, the maximum difference in magnitude between the velocity profiles is of $O(0.01)$. Similarly, the velocity fields predicted for the other modes shown in table $1, \boldsymbol{k}_{2}$ and $\boldsymbol{k}_{3}$, also agree within $O(0.01)$. Thus, despite the differences in formulation between the present study and that of McKeon \& Sharma (2010), the velocity structure associated with individual resolvent modes appears to be almost identical. However, the present analysis has the advantage that it also provides information regarding the pressure field associated with individual resolvent modes.

The variation in the magnitude of the pressure field is less pronounced (grey lines in figure $3 a, c$ ) than that of the velocity fields. There is a discernible peak in the pressure field near the critical layer for mode $\boldsymbol{k}_{b}$, and the magnitude of the pressure field decays above the critical layer. A peak in pressure is less evident for the longer mode $\boldsymbol{k}_{1}$, and the decay in the magnitude of the pressure field above the critical layer is slower. Similar to the wall-normal velocity, the phase of the pressure field does not vary significantly in the wall-normal direction (grey lines in figure $3 b, d$ ). Further, there is a near-constant $\pi / 2$ phase difference between the pressure and wall-normal velocity field. This means that the wall-normal velocity leads (lags) the pressure in space (time), as shown in figure 4.

The constant $\pi / 2$ phase difference between pressure and wall-normal velocity suggests that the primary contribution to the pressure field for the resolvent modes comes from the fast source term in the Poisson equation (2.13) such that $\nabla^{2} p_{k} \sim-\mathrm{i} k v_{k} U^{\prime}$. This is further supported by the Green's function solutions for the fast pressure shown in figure 3 (dashed grey lines) computed using (2.24). There is close agreement between the Green's function solution and the pressure obtained directly from the resolvent analysis for mode $\boldsymbol{k}_{b}$ (figure $3 a, b$ ), and the computed pressure fields are almost identical in phase and magnitude for mode $\boldsymbol{k}_{1}$ (figure $3 c, d$ ). Note that these observations pertaining to modes $\boldsymbol{k}_{b}$ and $\boldsymbol{k}_{1}$ are generally representative; pressure fields for the resolvent modes tend to be dominated by the linear, fast component. The reason for this dominance and the relative contributions of the slow and Stokes pressure are discussed further in $\S 3.3$.

Figure 4(a) shows the velocity and pressure structure associated with the mode $\boldsymbol{k}_{b}$ in physical space. As expected for Fourier modes, the velocity and pressure fields exhibit alternating positive and negative regions, dictated by the streamwise wavelength $\lambda_{x}^{+} \approx 190$. Consistent with the phase profiles shown in figure $3(b)$, isocontours of the wall-normal velocity (dashed black lines) and pressure (shading) remain upright and out of phase. The pressure field exhibits maxima in regions of increasing wall-normal 

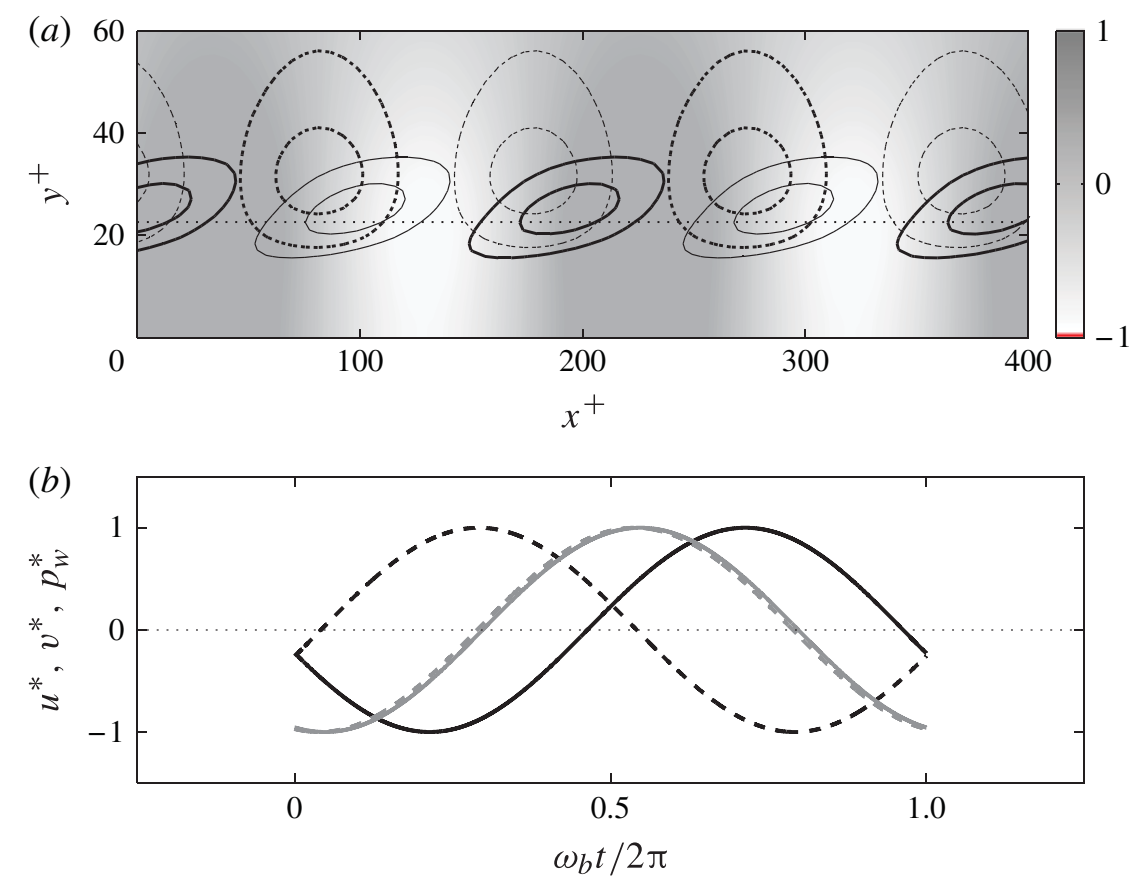

FIGURE 4. (a) Normalized contours of the streamwise velocity (solid lines), wall-normal velocity (dashed lines) and pressure field (shading) for mode $\boldsymbol{k}_{b}$. The bold lines denote positive velocities and the fine lines denote negative velocities. The contours are plotted at normalized velocity values $\pm 0.4, \pm 0.8$. (b) Temporal variation in the normalized wall-pressure field (solid grey line), and the streamwise (solid black line) and wall-normal velocities (dashed black line) at the critical layer, $y_{c}^{+}$. The dashed grey line shows the fast component of pressure.

velocity and minima in regions of decreasing wall-normal velocity, a consequence of the $\pi / 2$ phase difference between the fast pressure and the wall-normal velocity fields. The decrease in the phase of the streamwise velocity across the critical layer translates into velocity isocontours that lean in the streamwise direction (solid lines). Importantly, all of these observations are consistent with the spatial correlations for pressure and velocity presented in previous DNS and the experimental studies: correlation isocontours for streamwise velocity lean downstream (e.g. Marusic \& Heuer 2007; Guala, Metzger \& McKeon 2011), while isocontours for wall-normal velocity and pressure remain vertical (e.g. Kim 1989; Ghaemi \& Scarano 2013).

The conditionally averaged (variable-interval time averages around the passage of high-amplitude wall-pressure peaks) velocity and pressure measurements made by Johansson et al. (1987) at Reynolds number similar to that considered here $\left(\delta^{+} \approx 1800\right.$, where $\delta$ is boundary layer thickness) showed that high-amplitude peaks in wall pressure originated from velocity structures in the buffer region of the flow, with characteristic length scale $L^{+} \approx 150$ and speed propagation $c^{+} \approx 12$. Johansson et al. (1987) noted that positive wall-pressure peaks were associated with periods of increasing streamwise velocity and decreasing wall-normal velocity at $y^{+} \approx 15$. Figure $4(b)$, which shows the time-varying wall pressure and critical-layer velocities associated with the resolvent mode $\boldsymbol{k}_{b}\left(\lambda_{x}^{+} \approx 190, c^{+} \approx 12.5\right)$, reproduces these observations. Further, Johansson et al. (1987) found that the amplitude of the wall-pressure peaks scaled linearly with the velocities measured at $y^{+} \approx 15$, and therefore suggested that the linear, fast pressure contribution arising from the 
turbulence-mean shear interaction dominates the conditional averages. Again, this is broadly consistent with present results, which indicate that the fast component dominates the pressure field associated with the resolvent modes. Given these similarities, we suggest that velocity and pressure measurements made by Johansson et al. (1987) can be attributed to the presence of velocity structures resembling resolvent mode $\boldsymbol{k}_{b}$ in the real flow.

\subsection{Structure associated with individual resolvent modes}

Sharma \& McKeon (2013) show that the velocity fields associated with certain individual resolvent modes naturally give rise to hairpin-like structures. Here, we consider the relationship between such structures and the pressure field, providing further insight into the recent measurements of Ghaemi \& Scarano (2013), which show a bi-directional link between high-amplitude wall-pressure peaks and hairpin vortices.

Figure 5 shows the vortex structure and pressure associated with the wavenumberfrequency combination $\boldsymbol{k}_{b}$, summing contributions from both the left- and right-handed resolvent modes, $\boldsymbol{k}_{b}^{+}=(60,60,0.5)$ and $\boldsymbol{k}_{b}^{-}=(60,-60,0.5)$. Modes with positive $n$ create velocity structures that resemble right-handed helical propagating waves, and modes with negative $n$ create left-handed helical waves. Aside from this chirality, the resolvent modes are structurally identical. With just $+n$ or $-n$ modes, the velocity and pressure fields would align obliquely to the $x$ axis. Superposing both $\pm n$ leads to velocity and pressure fields that align in the streamwise direction.

The swirling strength (imaginary component of the complex-conjugate eigenvalue of the velocity gradient tensor, see Chakraborty, Balachandar \& Adrian 2005) isosurfaces for this mode combination presented in figure 5(a) show heads with alternate regions of prograde (i.e.in the direction of the mean shear) and retrograde azimuthal vorticity, along with legs aligned in the streamwise/wall-normal direction. Although the resolvent modes themselves generate an equal number of prograde and retrograde heads, Sharma \& McKeon (2013) show that the presence of the shear associated with the mean velocity profile suppresses the retrograde heads and strengthens the prograde heads, creating hairpin-like structures. This is illustrated in figure 5(b), which assumes that the amplitude of the mean velocity profile, $U$, is $100 \times$ that of the resolvent modes $\boldsymbol{k}_{b}^{+}$and $\boldsymbol{k}_{b}^{-}$. Figure 5(c) shows that the legs of the hairpin vortices are associated with counter-rotating motions in the azimuthal/wall-normal plane. The wall-normal velocity is positive immediately upstream of the hairpin heads $\left(n_{k} \theta / 2 \pi=0.5,1.5\right.$ in figure $\left.5 c\right)$. Figure $5(d)$ shows that sweeps $(u>0$ and $v<0, x^{+} \approx 150-200$ ) occur immediately downstream of the hairpin heads, while ejections ( $u<0$ and $v>0, x^{+} \approx 250-300$ ) occur immediately upstream of the hairpin heads.

Consistent with many previous observations (e.g. O'Farrell \& Martin 2009), the heads of the hairpin vortices lie above wall-pressure minima (figure $5 b$ ). This can be attributed to the $\pi / 2$ phase difference between the wall-normal velocity and pressure fields. The hairpin heads are associated with rapidly decreasing wall-normal velocity in $x$, which results in a pressure minimum (see e.g. $x^{+} \approx 100-150$ in figure $5 d$ ). The shear layer where the downward sweeps meet the upward ejections is associated with regions of positive pressure $\left(x^{+} \approx 220\right.$ in figure $\left.5 d\right)$. All of these structural features are consistent with the comprehensive recent measurements made by Ghaemi \& Scarano (2013), who show that positive peaks in wall pressure occur where the sweeps and ejections associated with the hairpin vortices meet, while negative wall-pressure events are associated with the vortex cores. 

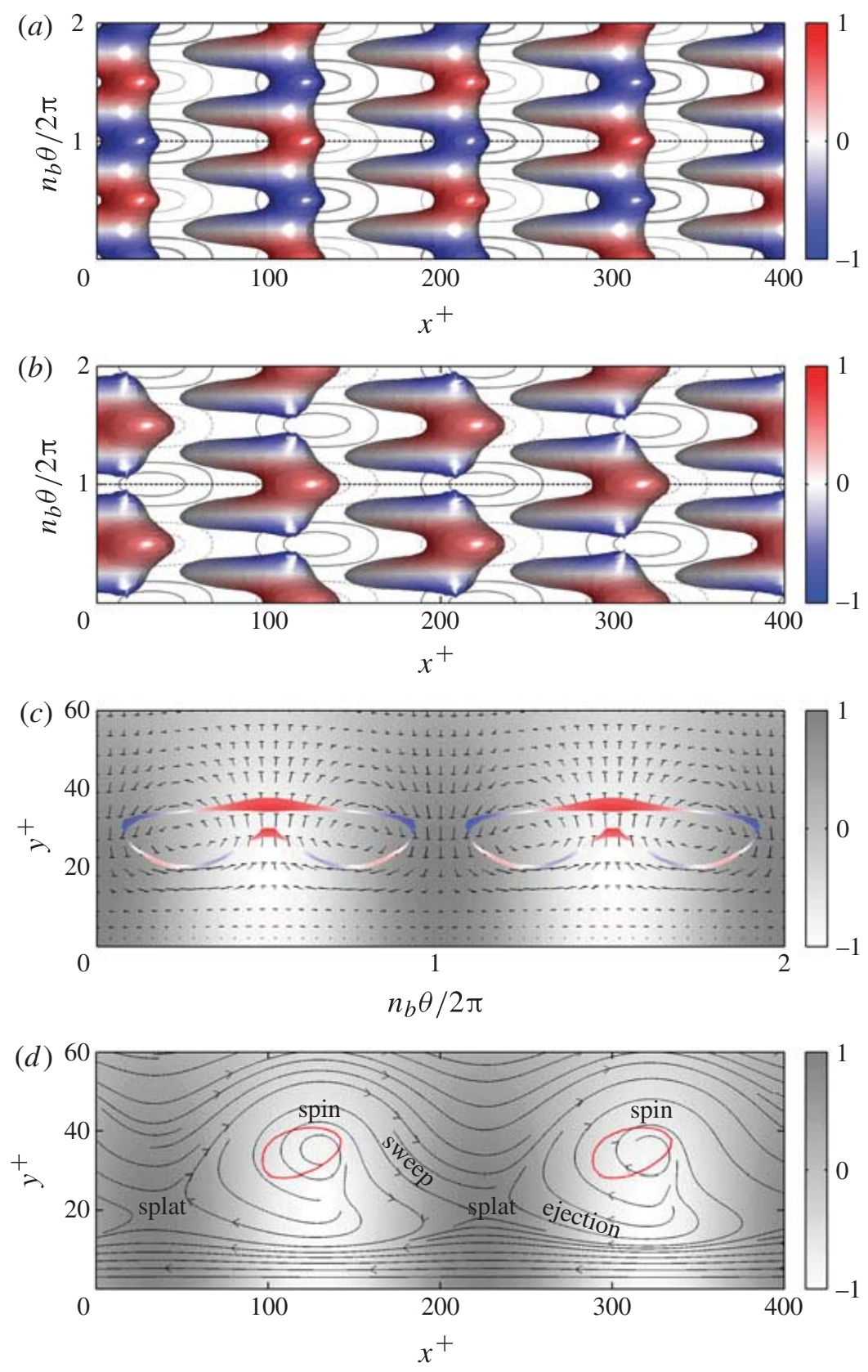

FigURE 5. $(a, b)$ Isosurfaces of constant swirling strength (at $50 \%$ of maximum) for mode $\boldsymbol{k}_{b}$ : (a) represents the resolvent mode alone, while $(b)$ includes the effect of the mean shear. The isosurfaces are shaded based on the local azimuthal vorticity. Red denotes prograde vorticity (i.e. in the direction of mean shear) and blue denotes retrograde vorticity. The contours beneath the swirl isosurfaces show the wall-pressure field. Bold contours show positive pressure while fine contours show negative pressure at normalized intervals $\pm(0.4,0.8)$. (c) Velocity, pressure and swirl in the azimuthal-wall-normal plane. The vectors show the local wall-normal and azimuthal velocities. The background shading shows the pressure field, and the red-blue shaded isosurfaces show the swirl field. Plot $(c)$ corresponds to streamwise location $x^{+}=0$ in $(b) .(d)$ Streamlines for mode $\boldsymbol{k}_{b}$, including the mean shear. Some streamlines have been left incomplete for clarity. Isocontours of constant swirling strength (at $50 \%$ of maximum) are shown in red. The background shading represents the pressure field. Plot $(d)$ corresponds to the azimuthal location $n_{b} \theta / 2 \pi=1$ in $(b)$. 
Note that the relationships between hairpin-like structures, wall-pressure peaks and the velocity fields discussed thus far (Johansson et al. 1987; O'Farrell \& Martin 2009; Ghaemi \& Scarano 2013) are observed only through conditional sampling or averaging of measurements. Instantaneous snapshots of the flow field do not show such clear relationships. In effect, the conditioning removes all the uncorrelated activity, leaving behind structure that is coherent. The results can then be interpreted in terms of the velocity and length scales that dominate the flow field for the specific conditioning event under consideration (e.g. high-amplitude pressure peaks). As discussed in Sharma \& McKeon (2013), the resolvent analysis effectively works in reverse: it predicts the structure associated with specified length and velocity scales.

The results presented in this section can also be interpreted in terms of the splat and spin interpretation of pressure suggested by Bradshaw \& Koh (1981). Specifically, Bradshaw \& Koh (1981) showed that the source terms for the pressure Poisson equation can be divided into two parts: one related to the square of the strain rate, and one related to the square of vorticity:

$$
\nabla^{2} p=\left(-S^{2}+\frac{1}{2}|\Omega|^{2}\right)
$$

Here, $S^{2}$ is the sum of squares of the principal rates of strain and $|\Omega|$ is the magnitude of the vorticity vector. Thus, negative source terms arise from the rate-of-strain contribution (splat), while positive source terms arise from the vorticity contribution (spin). Since $\nabla^{2} p$ is positive in regions of minimum pressure and negative in regions of maximum pressure, Bradshaw \& Koh (1981) concluded that the spin contribution leads to negative pressures and the splat contribution leads to positive pressures.

As noted earlier, the pressure fields obtained under the resolvent analysis for mode $\boldsymbol{k}_{b}$ correspond primarily to the fast pressure, with source term $-2\left(\partial v_{k} / \partial x\right) U^{\prime}(2.13)$. The mean shear, $U^{\prime}$, is positive and so regions with increasing wall-normal velocity in $x$ yield a splat contribution to pressure (negative source terms), while regions with decreasing wall-normal velocity yield a spin contribution (positive source terms). This distinction between the splat and spin contributions to pressure is illustrated by the streamline patterns shown in figure $5(d)$. As expected intuitively, the hairpin vortex cores generate a negative spin contribution to pressure, while the shear layers where the sweeps meet the ejections generate a positive splat contribution.

Finally, note that the streamlines shown in figure $5(d)$ resemble the well-known cat's eye patterns associated with two-dimensional, inviscid Kelvin-Stuart vortices. Sharma \& McKeon (2013) suggest that the resolvent modes may be interpreted as the threedimensional equivalent, albeit arising without an inflection point in the mean velocity profile and regularized due to viscosity.

\subsection{Pressure norm}

The dominance of the fast pressure for modes $\boldsymbol{k}_{b}$ and $\boldsymbol{k}_{1}$ observed in $\S 3.1$ suggests that the resolvent analysis pursued here generates forcing mode shapes that are near solenoidal, such that the slow source term in the pressure Poisson equation is almost zero, $\boldsymbol{\nabla} \cdot \boldsymbol{f}_{\boldsymbol{k}} \approx 0$ (2.25). This is supported by the observation that the normalized slow pressure contribution for mode $\boldsymbol{k}_{b}$ is of $O\left(10^{-9}\right)$ under the energy norm (figure 6). Unlike McKeon \& Sharma (2010) who projected the NSE onto divergence-free bases (thereby setting the slow pressure to exactly zero), we do not enforce solenoidal $f_{k}$ in this paper. Solenoidal forcing arises naturally from the singular value decomposition as the input direction that leads to the largest velocity response (i.e. highest $\sigma_{k}$ ) under 

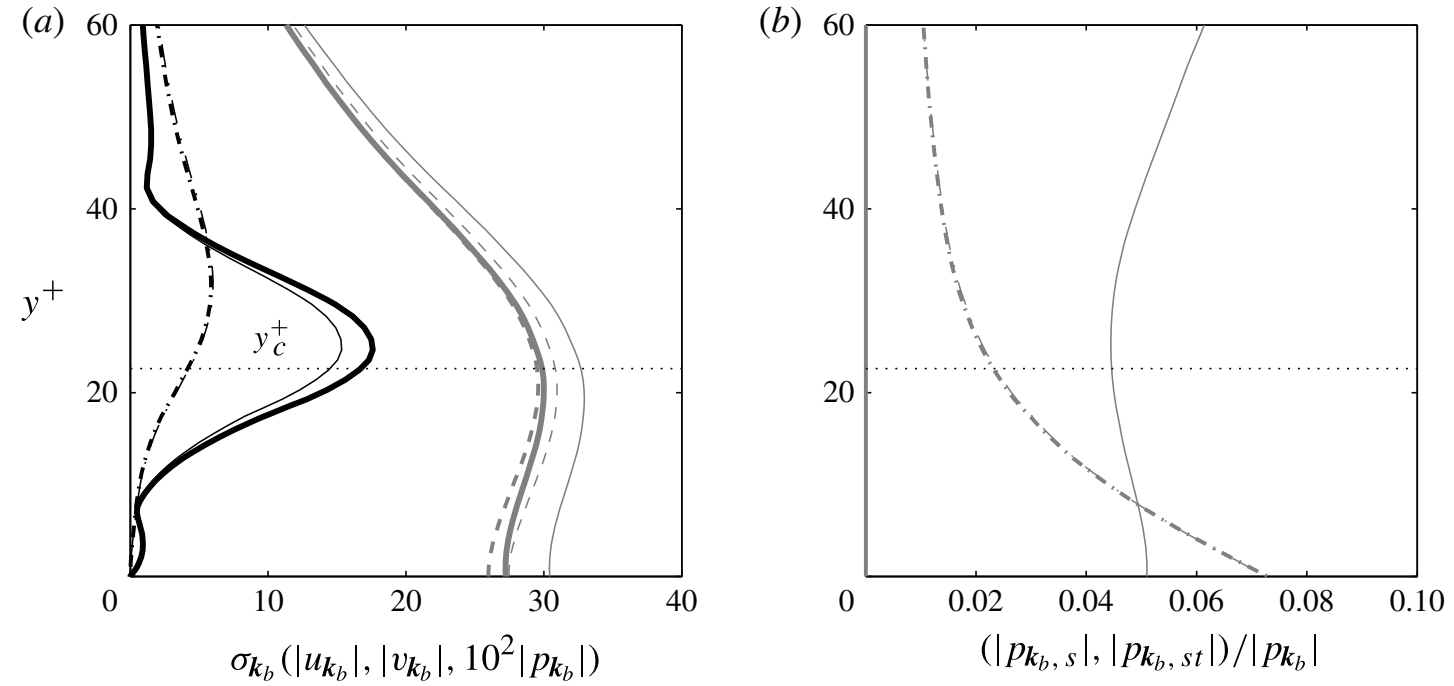

FIGURE 6. (a) Wall-normal variation in the magnitude of the pressure field (solid grey lines), and the streamwise (solid black lines) and wall-normal (dash-dotted lines) velocity fields. As before, the dashed grey lines denote the fast pressure. (b) Magnitude of the slow pressure field ( $p_{\boldsymbol{k}_{b}, s}$, solid lines) and Stokes pressure ( $p_{\boldsymbol{k}_{b}, s t}$ dash-dotted lines), normalized by the total pressure $\left(p_{\boldsymbol{k}_{b}}\right)$. In both panels, bold lines show predictions made under the energy norm and fine lines show predictions under the pressure norm. Note that the bold and fine dash-dotted lines in (a) and (b) overlap. The normalized slow pressure predicted under an energy norm is not visible in $(b)$ because it has magnitude of $O\left(10^{-9}\right)$.

an energy norm. Physically, this can be attributed to the Helmholtz decomposition (see e.g. Foias et al. 2001). Forcing that is not divergence-free does not contribute directly to the velocity field, and so a singular value decomposition under an energy norm (2.11) cannot identify non-solenoidal forcing as the most amplified input direction.

To see if the resolvent analysis yields different results under a pressure norm, we replace the scaling factor $\left[\begin{array}{ll}W_{u} & 0\end{array}\right]$ in (2.6) with $\left[\begin{array}{ll}0 & W_{p}\end{array}\right]$. This ensures that the orthonormality constraints on the singular response modes (2.10) translate to:

$$
\int_{0}^{1} p_{k, l}^{*} p_{k, m} r \mathrm{~d} r=\delta_{l m}
$$

such that the singular value decomposition identifies the forcing and response directions that yield the largest area-integrated fluctuating pressure.

Figure 6(a) compares the singular value-weighted velocity and pressure fields obtained under the energy norm for mode $\boldsymbol{k}_{b}$ (bold lines) with those obtained under the pressure norm, i.e. by weighting the intensity of the pressure fluctuations alone (fine lines). The velocity and pressure fields have been weighted by the singular values to ensure that we compare the responses for unit forcing across both norms (2.12). As expected, the magnitude of the pressure field increases under the pressure norm; the wall pressure increases by roughly $10 \%$, from 0.27 to 0.30 . The magnitude of the wall-normal velocity increases slightly under the pressure norm (by roughly $3 \%$ ), while the magnitude of the streamwise velocity decreases. Beyond these changes in magnitude, there are no significant changes in the structure of the velocity and pressure fields.

Note that the pressure norm yields a larger increase in pressure $(\approx 10 \%)$ than wall-normal velocity $(\approx 3 \%)$. Given the linear relationship between fast pressure and 
wall-normal velocity (2.24), this suggests that the slow pressure may play a larger role under the pressure norm. This is confirmed by the normalized pressure contributions shown in figure $6(b)$. Specifically, the normalized slow pressure contribution increases from $O\left(10^{-9}\right)$ under the energy norm, to approximately $5 \%$ under the pressure norm. This is comparable to the near-wall Stokes pressure contribution for this mode, but still much lower than the fast pressure contribution, which accounts for nearly $90 \%$ of the pressure field. Thus, even with a pure pressure norm, the fast component of pressure remains dominant in the present analysis. This is generally true for all the resolvent modes considered in this paper; the slow pressure contribution under a pressure norm remains lower than $5 \%$ for modes $\boldsymbol{k}_{1}, \boldsymbol{k}_{2}$, and $\boldsymbol{k}_{3}$ as well. In other words, the most amplified velocity and pressure fields appear to be relatively robust to norms weighting turbulent kinetic energy or pressure.

The above results also highlight an important distinction between the fast and slow component of pressure. While the fast pressure is directly linked to the turbulent velocity field via the source term (2.24), the slow pressure arises from the component of the nonlinear forcing term (2.25) that does not contribute to the velocity field. Thus, we expect correlations between the turbulent velocity and pressure field to be dominated by the fast component of pressure.

\section{Turbulence kernel}

Next, we consider the triadically consistent combination of modes $\boldsymbol{k}_{1}=(6, \pm 6,2 / 3)$, $\boldsymbol{k}_{2}=(1, \pm 6,2 / 3)$, and $\boldsymbol{k}_{3}=(7, \pm 12,2 / 3)$ listed in table 1 . Sharma \& McKeon (2013) show that this limited set of modes can be thought of as a turbulence kernel that (i) generates realistic structure consisting of modulating hairpin packets and (ii) captures key features of the interaction between the large- and small-scale streamwise velocity in the overlap region of the flow. Here, we extend this kernel to include pressure.

To arrive at the results discussed in this section, the complex amplitudes for the velocity and pressure fields for the three resolvent modes were set to $\left(a_{1}, a_{2}, a_{3}\right)=$ $(1,4.5,-0.83 i)$, and the amplitude of the mean velocity profile was set to 1000 , i.e. the total velocity field for the turbulence kernel is $\tilde{\boldsymbol{u}}=1000 U+a_{1} \boldsymbol{u}_{k_{1}}+a_{2} \boldsymbol{u}_{\boldsymbol{k}_{2}}+$ $a_{3} \boldsymbol{u}_{k_{3}}$, where $\boldsymbol{u}_{\boldsymbol{k}_{1}}$ etc., represent the energy-normalized resolvent modes (2.11). The total pressure field can be obtained via a similar linear summation. Note that the amplitudes $\left(a_{1}, a_{2}, a_{3}\right)$ were chosen to be representative of previous observations.

Figure 7 shows the spatial structure and wall-pressure field associated with this mode combination. The swirl field (shaded isosurfaces) shows discrete packets of prograde hairpin-like structures. Sharma \& McKeon (2013) provide a detailed description of this structure in terms of the beating patterns arising from the interaction of Fourier modes. The extended kernel considered here shows that the wall-pressure field retains a clear imprint of the hairpin-like structures populating the flow field. Consistent with the results presented in the previous sections, the hairpin heads are associated with negative wall pressure, while the regions between the hairpins are associated with positive wall-pressure (along line $A^{\prime}-A^{\prime}$ in figure 7).

Figure 8 shows the streamwise velocity at the critical layer $\left(y_{c}^{+} \approx 110\right)$, the wall pressure, and the vortex structure for a slice through the turbulence kernel shown in figure 7. The streamwise velocity field is dominated by the longer $\boldsymbol{k}_{2}$ mode (i.e. with streamwise length scale $L^{+}=2 \pi R^{+} / 1 \approx 11000$, figure $8 a, b$ ), while the wall-pressure field is dominated by structures corresponding to shorter modes $\boldsymbol{k}_{1}$ and $\boldsymbol{k}_{3}\left(L^{+} \approx 1600-2000\right.$, figure $\left.8 c, d\right)$. This discrepancy in length scale can be attributed to the form of the resolvent modes. Sharma \& McKeon (2013) show that 

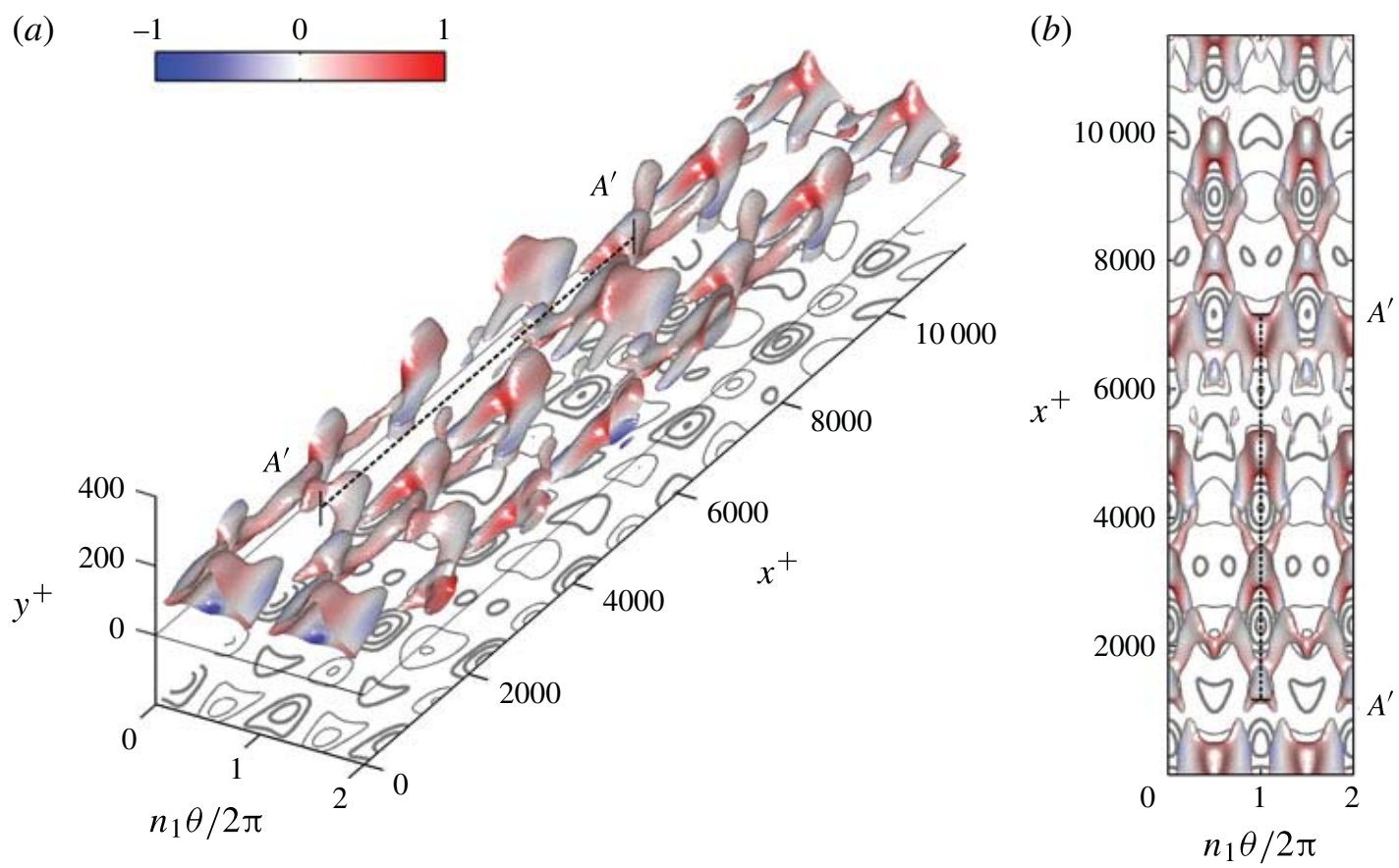

FIGURE 7. Isosurfaces of constant swirling strength (at $50 \%$ of maximum) for the turbulence kernel comprising modes $\boldsymbol{k}_{1}, \boldsymbol{k}_{2}$, and $\boldsymbol{k}_{3}$. The isosurfaces are shaded based on the local azimuthal vorticity. Red denotes prograde rotation, and blue denotes retrograde rotation. The contours beneath the isosurfaces represents the normalized wall-pressure field at intervals $\pm(0.3,0.6,0.9)$. Bold contours show positive pressure, fine contours show negative pressure. (a) Isometric view, $(b)$ plan view.

the magnitude of the streamwise velocity is comparable for all three resolvent modes, $\boldsymbol{u}_{k_{1}}, \boldsymbol{u}_{k_{2}}$, and $\boldsymbol{u}_{\boldsymbol{k}_{3}}$. As a result, the longer $\boldsymbol{u}_{\boldsymbol{k}_{2}}$ mode dominates the flow field due to its larger amplitude, $a_{2}>\left(a_{1}, a_{3}\right)$. In contrast to the streamwise velocity, the wall-normal velocity is approximately 4-5 times larger for the more circular resolvent modes, $\boldsymbol{u}_{\boldsymbol{k}_{1}}$ and $\boldsymbol{u}_{k_{3}}$. Coupled with the fact that $\left(k_{1}, k_{3}\right)>k_{2}$, this means that the fast pressure source term $\propto k v_{k} U^{\prime}$ is much larger for $\boldsymbol{u}_{k_{1}}$ and $\boldsymbol{u}_{k_{3}}$. As a result, the wall-pressure field is dominated by these smaller, circular structures (figures $7,8 c, d$ ). Note that these limited observations are broadly consistent with the DNS of Jimenez \& Hoyas (2008), who show that the streamwise length scales associated with the pressure spectrum tend to be smaller than those associated with the streamwise velocity spectrum. We consider spectral features of the wall-pressure field in greater detail in the following section.

The large-eddy simulation of Chung \& McKeon (2010) and experimental observations of Jacobi \& McKeon (2013) show that the apparent amplitude modulation effect observed in recent studies (e.g. Marusic et al. 2010) can also be interpreted in terms of the phase relationship between the large-scale streamwise velocity and the envelope of the small-scale activity. Specifically, Chung \& McKeon (2010) and Jacobi $\&$ McKeon (2013) show that the envelope of the small-scale velocity is in phase with the large-scale streamwise velocity close to the wall, and that the phase difference increases away from the wall, passing through $\pi / 2$ in the overlap region. Based on these studies, Sharma \& McKeon (2013) chose the complex amplitudes of the three resolvent modes such that the turbulence kernel is representative of the smalland large-scale interaction in the overlap region, i.e. the envelope of the small scales 

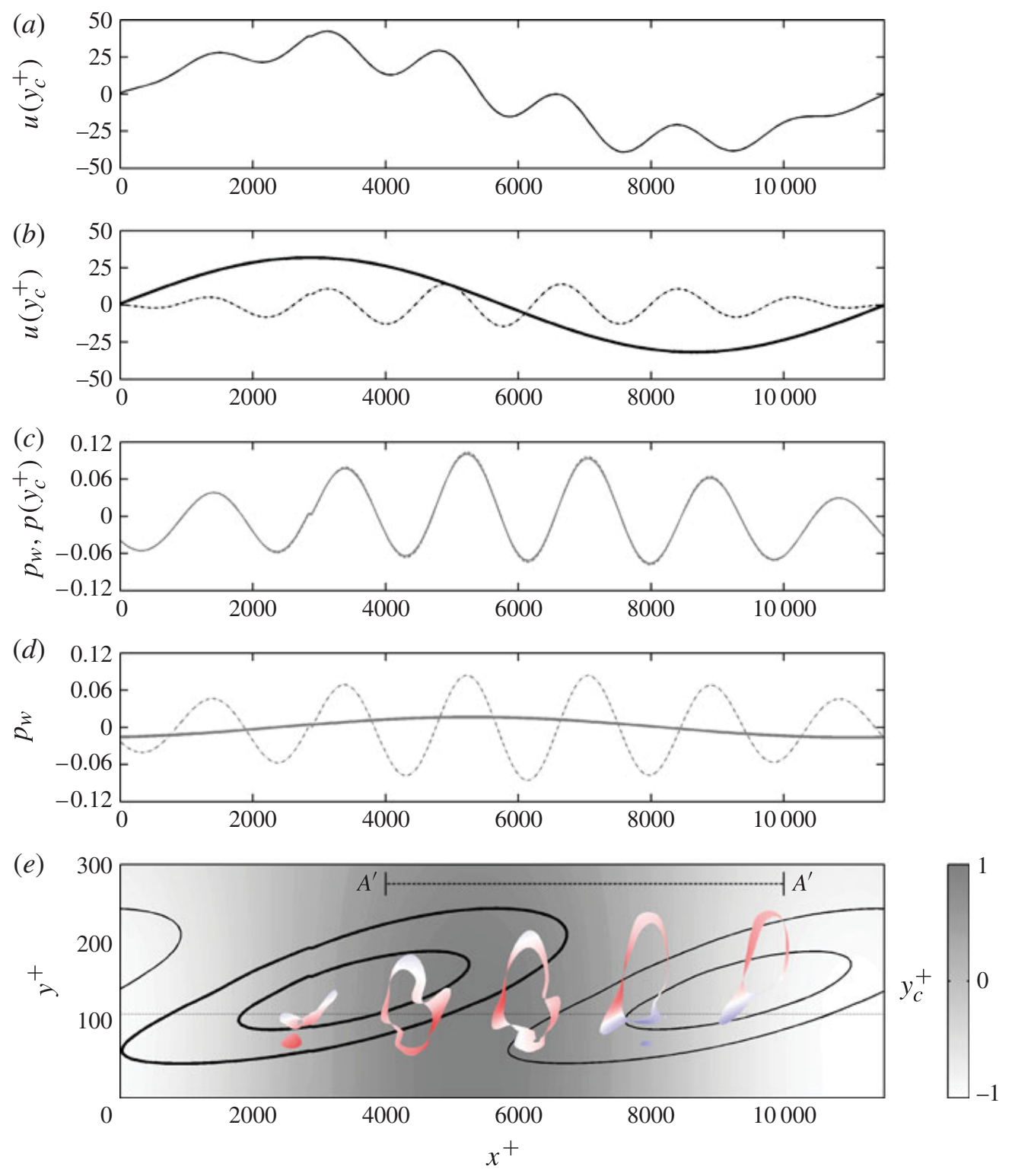

FIGURE 8. (a) Variation in the streamwise velocity at the critical layer for the turbulence kernel comprising modes $\boldsymbol{k}_{1}, \boldsymbol{k}_{2}$, and $\boldsymbol{k}_{3}$. (b) Streamwise velocity contributions from the large-scale $\boldsymbol{k}_{2}$ mode (bold line) and the small-scale $\boldsymbol{k}_{1}, \boldsymbol{k}_{3}$ modes (dashed-dotted line) at the critical layer. (c) Variation in the pressure field for the turbulence kernel. Solid lines represent the wall pressure, $p_{w}$, while dashed lines represent the pressure at the critical layer, $p\left(y_{c}^{+}\right)$. Note that $p_{w}$ and $p\left(y_{c}^{+}\right)$are practically indistinguishable. $(d)$ Wall-pressure contribution from the large-scale $\boldsymbol{k}_{2}$ mode (bold line) and the smaller scale $\boldsymbol{k}_{1}, \boldsymbol{k}_{3}$ modes (dash-dotted line). (e) Slice through the swirling strength isocontours shown in figure 7 , at location $n_{1} \theta / 2 \pi=1$. The line $A^{\prime}-A^{\prime}$ is consistent across figures 7 and 8 . The background shading shows the normalized large-scale pressure field and the contours show the normalized large-scale streamwise velocity field at intervals $\pm(0.4,0.8)$. The bold lines denote positive velocity, the fine lines denote negative velocity.

leads the large-scale streamwise velocity by $\pi / 2$. As illustrated in figure $8(b)$, this means that the small-scale streamwise velocity (dashed-dotted line) is most energetic in regions of decreasing large-scale $u$ at the critical layer (bold line). 
The present study shows that the interaction between the large and small scales leads to an apparent amplitude modulation effect in the wall pressure as well. Like the small-scale streamwise velocity, the small-scale wall pressure is most energetic in the middle of the plotting window, $x^{+} \approx 4000-8000$ in figure $8(d)$, where the hairpin vortices are concentrated (figure $8 e$ ). This coincides with the peak in large-scale wall pressure (figure $8 d$, bold line). Thus, unlike the streamwise velocity at the critical layer (figure $8 b$ ), the envelope of the small-scale activity in wall pressure is in phase with the large-scale wall pressure (figure $8 d$ ). The $\pi / 2$ difference in the phase relationships between the large and small scales for streamwise velocity and pressure can be attributed to the form of the resolvent modes: the streamwise velocity at the critical layer lags the pressure field by $\pi / 2$ in $x$ (figure 8 , see also figure 4).

Finally, note that the $\pi / 2$ difference in phase relationships for streamwise velocity and wall pressure is consistent with the observations of Thomas \& Bull (1983). As discussed earlier, Thomas \& Bull (1983) found that the envelope of the high-frequency wall-pressure fluctuations was approximately $\pi / 2$ out of phase with the low-frequency wall-pressure fluctuations. In contrast, the envelope of the high-frequency wall shear stress was observed to be nearly in phase with the low-frequency wall shear stress. Closer to the wall, the envelope of the small-scale activity is expected to be in phase with the local large-scale streamwise velocity (Chung \& McKeon 2010; Jacobi \& McKeon 2013). In other words, closer to the wall, we expect increased small-scale activity in regions with high large-scale streamwise velocity and wall shear stress (e.g. at $x \approx 2000$ in figure $8 e$ ). In agreement with the large- and small-scale interdependence observed by Thomas \& Bull (1983), this would lead to the envelope of the small-scale wall pressure lagging the large-scale pressure by roughly $\pi / 2$ in $x$.

\section{Spectral predictions}

A complete spectral description within the resolvent analysis framework requires knowledge of the nonlinear forcing $f_{k}$ present in the flow at each wavenumberfrequency combination. The singular values $\sigma_{k}$ can be interpreted as the filters that determine how this forcing translates into velocity and pressure responses; wavenumber-frequency combinations that are highly amplified (high $\sigma_{k}$ ) are more energetic in the flow. Thus, the velocity and pressure spectra reflect both the magnitude of the nonlinear forcing present in the flow at each $k$ and the amplification/filtering effect of the singular values. A recent study by Moarref et al. (2013) demonstrates that this filtering effect plays a key role in shaping the streamwise velocity spectrum. With the simplest possible broadband forcing model (i.e. unit forcing in the direction of $\boldsymbol{f}_{\boldsymbol{k}}$ at each $\boldsymbol{k}$ such that the velocity field for that wavenumber-frequency combination is given by $\sigma_{k} \boldsymbol{u}_{k}$ ), Moarref et al. (2013) qualitatively reproduce many features of the streamwise velocity spectrum, including the presence of a near-wall peak that scales with inner units, and the appearance of an additional peak in the logarithmic region of the flow at high Reynolds number that scales with outer variables.

In this section, we employ the broadband forcing assumption to consider the differences between velocity and pressure spectra $(\$ 5.1)$, and to study the scale dependence of the wall-pressure propagation speed (\$5.2). By comparing the spectral predictions with previous DNS results (Jimenez \& Hoyas 2008), we also identify where the nonlinear forcing is more important in shaping the spectra than the filtering due to $\sigma_{k}$. 
Under the broadband forcing assumption, the relative amplitudes of the resolvent modes at each $\boldsymbol{k}$ are set simply by the singular values, $\sigma_{\boldsymbol{k}}$ (see (2.12)). Thus, the premultiplied spectral densities for the velocity and pressure fields become:

$$
\left[\begin{array}{c}
E_{u u}(k, n, y) \\
E_{v v}(k, n, y) \\
E_{w w}(k, n, y) \\
E_{p p}(k, n, y)
\end{array}\right]=k n \int_{-\infty}^{\infty} \sigma_{k}^{2}\left[\begin{array}{c}
\left|u_{k}(y)\right|^{2} \\
\left|v_{k}(y)\right|^{2} \\
\left|w_{k}(y)\right|^{2} \\
\left|p_{k}(y)\right|^{2}
\end{array}\right] \mathrm{d} \omega .
$$

The spectral predictions shown below were obtained by evaluating (5.1) over the ranges: $k=(0.01-316), n= \pm(0-316)$, and $c=\omega / k=0.1-1.0$ at Reynolds number $R e=75000\left(R^{+}=1800\right)$. These ranges correspond to modes with streamwise wavelength $\lambda_{x} \approx 0.02 R-630 R$, and wave speed ranging from $10 \%$ to $100 \%$ of the pipe centreline velocity, or $\lambda_{x}^{+} \approx 36-10^{6}$ and $c^{+} \approx 2.5-25$. Wavenumber-frequency combinations outside of these ranges are not energetically important (low $\sigma_{k}$ ), and therefore do not change the results appreciably.

Keep in mind that the simple model developed here can only predict second-order statistics such as power spectra, r.m.s. intensities and turbulent kinetic energy, which do not require knowledge of the interaction across spectral space. Higher-order statistics such as skewness and kurtosis also require information regarding the relative amplitude and phase of the different Fourier resolvent modes, which depends on the amplitude and phase of the nonlinear forcing present in the flow.

Further, note that the analysis pursued here is in many ways complementary to the structure-based model for wall pressure developed by Ahn et al. (2010) within the broad attached-eddy framework. Ahn et al. (2010) superpose contributions from individual eddies of a prescribed hairpin-like shape, with a number density that yields total circulation consistent with the mean velocity profile, to predict spectra and spatial correlations for the wall-pressure field. In the present study, contributions are superposed from the Fourier resolvent modes, which represent distinct flow structures, identified directly from the NSE. Both models require knowledge of the mean velocity profile and both assume that the linear, fast component of pressure dominates. Reconciling the two approaches (e.g. using resolvent modes in the attached-eddy framework) may be a fruitful avenue for further research.

\subsection{Spectral content}

Figure $9(a)$ shows the pre-multiplied spectra for streamwise velocity (solid line), wall-normal velocity (dashed line) and pressure (grey line) at $y / R=0.1$ obtained under broadband forcing, i.e. the simple singular value-weighted integrals of the resolvent modes shown in (5.1). Also shown are the pre-multiplied spectra for streamwise velocity (dark grey shading) and pressure (light grey shading) at a location in the buffer region of the flow, $y^{+}=15$. In general, the spectra suggest that the streamwise velocity fluctuations originate from structures that are much longer than they are wide $\left(\lambda_{x}>\lambda_{\theta}\right)$, while the structures that contribute to the pressure and wall-normal velocity are more circular in terms of aspect ratio. Further, figure $9(a)$ shows that the length scales associated with the streamwise velocity and pressure spectra increase with distance from the wall. The peak in wall-pressure spectrum moves from $\left(\lambda_{x}, \lambda_{\theta}\right)=(0.04,0.06)$ at $y^{+}=15$ to $\left(\lambda_{x}, \lambda_{\theta}\right)=(0.4,0.7)$ at $y / R=0.1$. The peak in the streamwise velocity spectrum moves from $\left(\lambda_{x}, \lambda_{\theta}\right)=(0.16,0.03)$ at $y^{+}=15$ to $\left(\lambda_{x}, \lambda_{\theta}\right)=(7.2,0.4)$ at $y / R=0.1$. These trends are broadly similar to the DNS results obtained by Jimenez \& Hoyas (2008) for channel flow at a Reynolds 

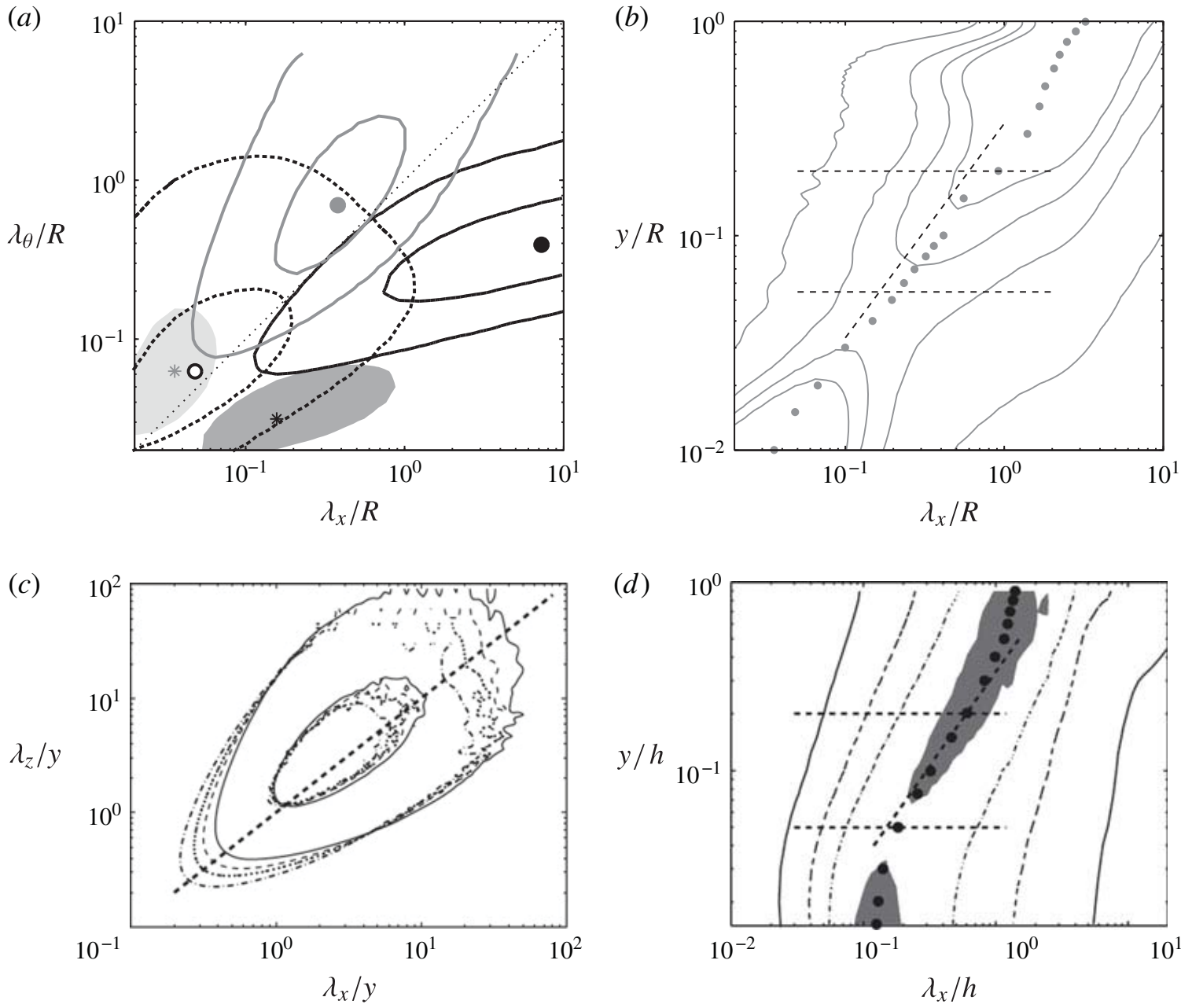

FIGURE 9. (a) Pre-multiplied spectra for streamwise velocity (solid black lines), wall-normal velocity (dashed black lines), and pressure (grey lines) at $y / R=0.1$ predicted under a broadband forcing assumption. Contour lines are plotted at normalized values $(0.125,0.625)$. The shaded regions show the normalized spectra $(>0.625)$ for streamwise velocity (dark grey) and for pressure (light grey) at $y^{+}=15$. The azimuthal wavenumber is estimated as $\lambda_{\theta} / R=2 \pi / n$. (b) Spectral density for pressure as a function of $\lambda_{x} / R$ and $y / R$. Contours represent normalized pressure at (0.03 (0.09) 0.30$)$. The dashed horizontal lines represent $y^{+}=100$ and $y / R=0.2$, and the dashed diagonal line is $\lambda_{x}=3 y$. In both plots, the point markers denote maxima. For comparison, figure 9 from Jimenez \& Hoyas (2008) showing the spectral densities for pressure in turbulent channel flow at $R e_{\tau}=u_{\tau} h / v=2000$ ( $h$ is the channel half-height) is reproduced in $(c)$ and $(d):(c)$ shows pressure spectra at $y / h=0.1$ (solid lines), 0.15 (dashed lines), 0.20 (dotted line), and 0.30 (dash-dotted lines).

number similar to that considered here $\left(R e_{\tau}=2000\right)$, which is encouraging given the simplicity of the rank-1 and broadband forcing assumptions.

Of course, there are some quantitative differences between the present predictions and the DNS results. For instance, wall-normal velocity spectrum peaks at $\left(\lambda_{x}, \lambda_{\theta}\right)=$ $(0.05,0.06)$ at $y / R=0.1$ under the broadband forcing assumption, while the DNS predicts larger outer-normalized length scales, $\approx 0.1 h-0.2 h$. Further, it is well known that the peak in streamwise velocity at $y^{+}=15$ falls at the length scales corresponding 
to the energetic near-wall cycle, $\left(\lambda_{x}^{+}, \lambda_{\theta}^{+}\right) \approx(1000,100)$. In contrast, the broadband forcing assumption predicts a peak at $\left(\lambda_{x}, \lambda_{\theta}\right)=(0.16,0.03)$, or $\left(\lambda_{x}^{+}, \lambda_{\theta}^{+}\right) \approx(300,50)$.

Consistent with figure $9(a)$, the predicted spectral density for pressure (figure $9 b$ ) suggests the presence of two distinct peaks: a near-wall peak at $y / R<0.03\left(y^{+}<60\right)$ comprising small-scale structures with $\lambda_{x}<0.1 R\left(\lambda_{x}^{+}<180\right)$, and an outer peak at $y / R>0.1$ comprising large-scale structures with $\lambda_{x} \sim O(R)$. Further, there is a near-linear relationship $\lambda_{x} \sim y$ in the $\log$ region of the flow. These predictions are again similar to the DNS results of Jimenez \& Hoyas (2008) (figure 9c,d). The major differences between the DNS results and the present predictions are concentrated in the near-wall region and close to the centreline. The present results suggest a continuous decrease in $\lambda_{x}$ with $y$ close to the wall. In contrast, the DNS results show that the peak in spectral density falls at a roughly constant streamwise wavelength $\lambda_{x} \approx 0.1 h$ near the wall. Further, the DNS results exhibit near-vertical spectral density contours close to the centreline, while the present predictions show sharp gradients for $y / R>0.5$. Some of the differences close to the centreline could be attributed to differences in geometry (pipe versus channel). However, in general these results suggest that the rank-1 and broadband forcing assumptions must be evaluated for the smaller, slower-moving modes localized near the wall and for the larger modes close to the pipe centreline, which is also consistent with the observations of Moarref et al. (2013). An alternative (albeit complementary) interpretation is that the gain-based decomposition and mode ordering pursued in this paper are less well suited for capturing the dynamics near the wall and close to the centreline. These issues will be explored further in future work.

\subsection{Wall-pressure propagation speed}

Despite significant evidence suggesting that the propagation speed for wall pressure is scale-dependent, most experimental studies are limited to employing a single propagation speed (i.e.integrated over all length scales) due to practical constraints. In this section, we employ the resolvent analysis to provide further insight into the scale-dependence of wall-pressure propagation speeds. For brevity, we limit the discussion to modes that are circular in aspect ratio (i.e. modes with equal streamwise and azimuthal wavenumbers, $k=n$ ), which are known to dominate the wall-pressure field (a result also captured by the broadband forcing assumption, figure $9 a$ ). Further, we continue with the rank-1 and broadband forcing assumptions employed in the previous section.

Figure 10 shows the wall-normal velocity and pressure fields for resolvent modes with the same streamwise and azimuthal wavenumber as mode $\boldsymbol{k}_{b}, k=n=60$, but with varying propagation speed, $c^{+}=0-25$, at Reynolds number $R e=75000$. Per McKeon \& Sharma (2010) and McKeon et al. (2013), for slower-moving modes with $c^{+}<10$, the wall-normal velocity is localized at a near-constant elevation $y^{+} \approx 10$ (figure 10a). However, as the speed increases above $c^{+}>10$, the wall-normal velocity begins to localize around the critical layer where the mean velocity matches the mode speed (solid black line). For $c^{+}>15$, the modes do not have a significant velocity presence near the wall. McKeon et al. (2013) term the slower moving modes with $c^{+}<10$ as attached to the wall. The modes with intermediate speed $c^{+}=10-15$ are considered attached and critical. The faster moving modes with $c^{+}>15$ that do not have a significant presence at the wall are considered to be detached and critical.

The analysis developed here shows that the pressure fields associated with the attached modes extend to the wall, while the detached-critical modes with $c^{+}>15$ 


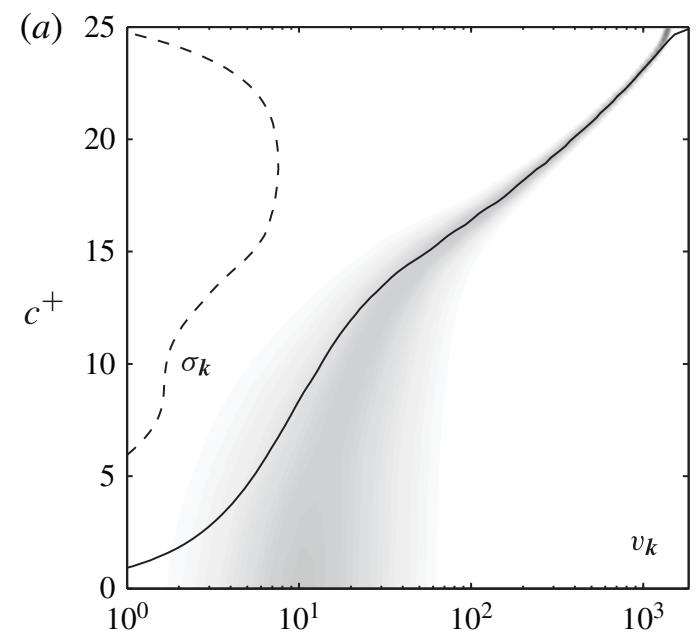

(b)
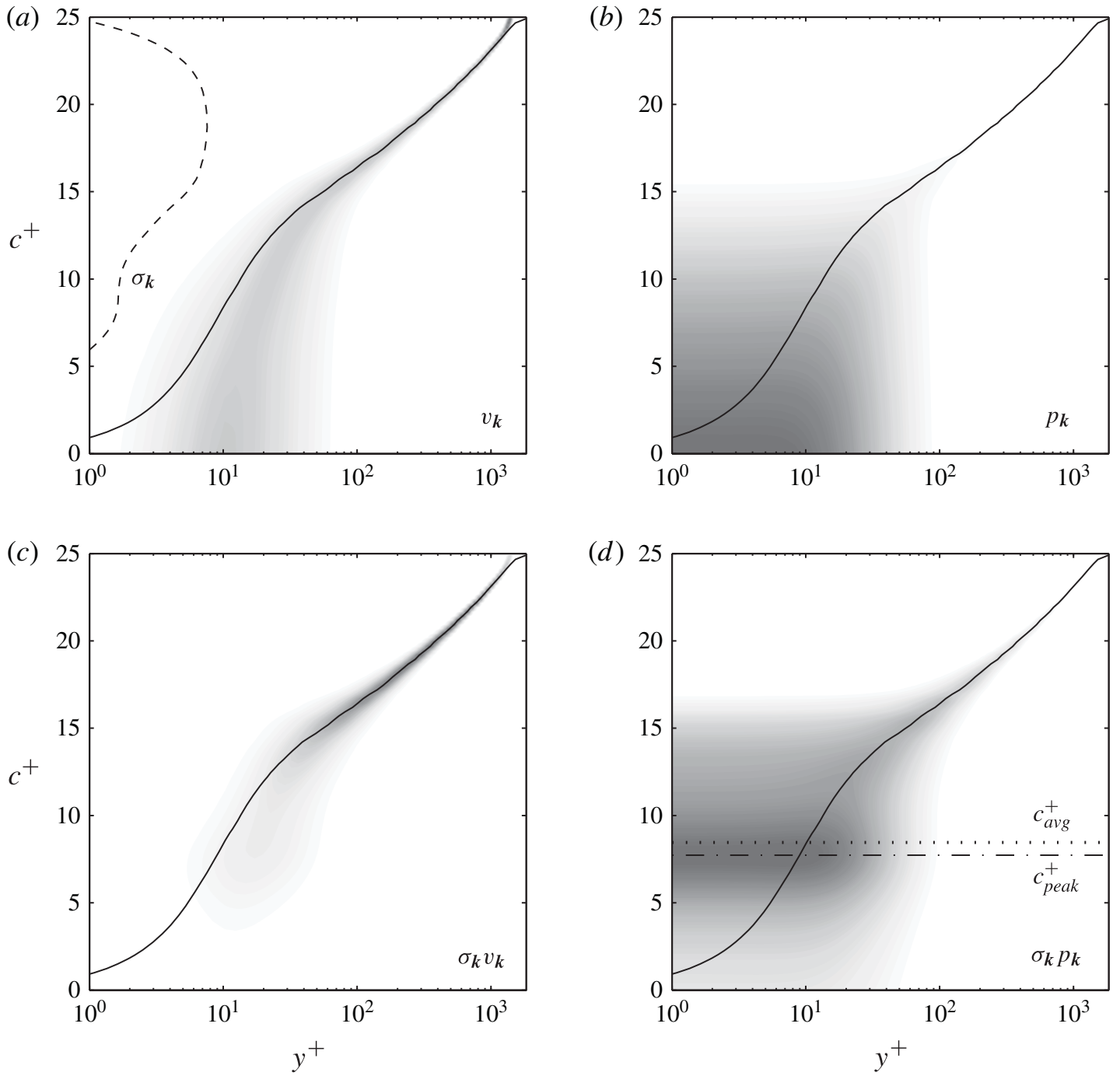

FIGURE 10. Wall-normal distribution of pressure and velocity for modes with $k=n=60$ at $R^{+}=1800\left(\lambda_{x}^{+}=\lambda_{\theta}^{+} \approx 190\right)$. $(a, b)$ The distribution of the normalized velocity $\left(v_{k}\right)$ and pressure field $\left(p_{k}\right)$ for varying speed $c^{+} .(c, d)$ The amplification-weighted velocity $\left(\sigma_{k} v_{k}\right)$ and pressure distributions $\left(\sigma_{k} p_{k}\right)$. In all plots, the solid black line represents the mean velocity profile. The dashed line in $(a)$ shows the variation in singular values with wave speed. Horizontal lines in $(d)$ show the propagation speed corresponding to the peak in wall pressure, $c_{\text {peak }}^{+}$, and a weighted average, $c_{a v g}^{+}$.

do not have a significant wall-pressure signature (figure 10b). Further, the strength of the pressure field decreases with increasing mode speed. These trends can be attributed to the structure of the Green's function (2.22) associated with these modes, and the form of the fast source terms in the pressure Poisson equation $\left(\propto k v_{k} U^{\prime}\right)$. Specifically, figure 2(b) shows that the Green's function for wall pressure does not have a long range for modes with $k=n=60$ (mode $\boldsymbol{k}_{b}$ ), decaying by two orders of magnitude over wall-normal distance $y \approx 0.05\left(y^{+} \approx 100\right)$. Slower-moving attached modes with wall-normal velocities localized closer to the wall have pressure sources located within the range of the Green's function. In addition, the source terms for the attached modes are stronger because such modes are localized in regions with high mean shear. Since the wall pressure is given by the integral of the source 
terms weighted by the Green's function (2.24) and (2.25), such slower-moving modes have much larger wall pressures. For critical modes with $c^{+}>15$, the wall-normal velocity (and hence pressure source) is localized above $y^{+}>100$, where the mean shear is lower and the Green's function is negligible. As a result, such modes do not contribute much to the wall pressure.

Keep in mind that the results shown in figure $10(a, b)$ are normalized. Under the broadband forcing assumption (5.1), the magnitude of the unweighted velocity and pressure fields must be weighted by the singular values. The weighted profiles $\sigma_{k} v_{k}$ and $\sigma_{k} p_{k}$ are shown in figures $10(c)$ and $10(d)$, respectively. In general, the singular values $\sigma_{k}$ increase with increasing speed for $c^{+}<20$ (dashed line, figure 10a). As a result, the weighted wall-pressure field exhibits a trade-off between increasing singular value with $c^{+}$and decreasing source strength/wall proximity. While the normalized wall pressure was largest for the slowest modes (figure 10b), the weighted wall pressure peaks near $c_{\text {peak }}^{+}=7.7$ (figure $10 d$ ). This means that, for wavelength $\lambda_{\theta}^{+} \approx \lambda_{x}^{+}=190$, velocity structures moving with speed $c_{\text {peak }}^{+}=7.7$ have the largest wall-pressure signature under the broadband forcing assumption employed here. Figure $10(d)$ also shows an average propagation speed (del Alamo \& Jimenez 2009), defined as:

$$
c_{\text {avg }}^{+}=\frac{\int_{0}^{U_{C L}^{+}} c^{+} \sigma_{k} p_{k} \mathrm{~d} c^{+}}{\int_{0}^{U_{C L}^{+}} \sigma_{k} p_{k} \mathrm{~d} c^{+}},
$$

which is $c_{\text {avg }}^{+}=8.5$ for this wavenumber-frequency combination. Note that these propagation speeds coincide with a brief plateau in $\sigma_{k}$ starting at $c^{+} \approx 8$ (figure $10 a$, dashed line).

Figure 11 shows velocity and pressure fields for larger modes, $k=n=20\left(\lambda_{\theta}^{+} \approx\right.$ $\lambda_{x}^{+}=570$ ). Despite a three-fold increase in streamwise and azimuthal length scale, these larger modes exhibit similar behaviour to the modes shown in figure 10, i.e. the strength of the wall-pressure signature is determined by a trade-off between wall proximity and singular value. For the larger modes shown in figure 11, the pressure and velocity fields have a greater wall-normal extent. As a result, the transition to detached-critical behaviour occurs at a slightly higher speed, $c^{+} \approx 17$ (figure $11 a, b$ ). Also, these larger modes have higher singular values compared to the $k=n=60$ modes considered in figure 10. For example, the singular value increases from $\sigma_{k}=5$ (figure $10 a$, dashed line) to $\sigma_{k}=20$ (figure $11 a$, dashed line) for $c^{+}=15$. This increase in amplification results in a peak propagation speed, $c_{\text {peak }}^{+}=15$ (figure $11 d$ ), that is nearly twice that of the smaller $k=n=60$ modes. Thus, for wavelength $\lambda_{\theta}^{+} \approx \lambda_{x}^{+}=570$, velocity structures in the log region of the flow have the largest wall-pressure signature under broadband forcing. Note that the increase in the average propagation speed is less dramatic, from $c_{\text {avg }}^{+}=8.5$ for the modes shown in figure 10 to $c_{\text {avg }}^{+}=11$ for the modes shown in figure 11 .

Figure 12 shows the peak and average wall-pressure propagation speed as a function of wavelength for circular modes $\left(\lambda_{x}^{+}=\lambda_{\theta}^{+}\right)$across a range of Reynolds number $R e=5000-233000\left(R^{+}=180-5000\right)$. As intuitively expected, the propagation speeds increase with increasing length scale. In other words, wall-pressure structures with longer streamwise and azimuthal length scales are more likely to originate from faster-moving velocity structures further away from the wall. At all Reynolds numbers, the peak propagation speed jumps from $c_{\text {peak }}^{+} \approx 8$ for $\lambda_{x}^{+}<400$ to $c_{\text {peak }}^{+} \approx 15$ 


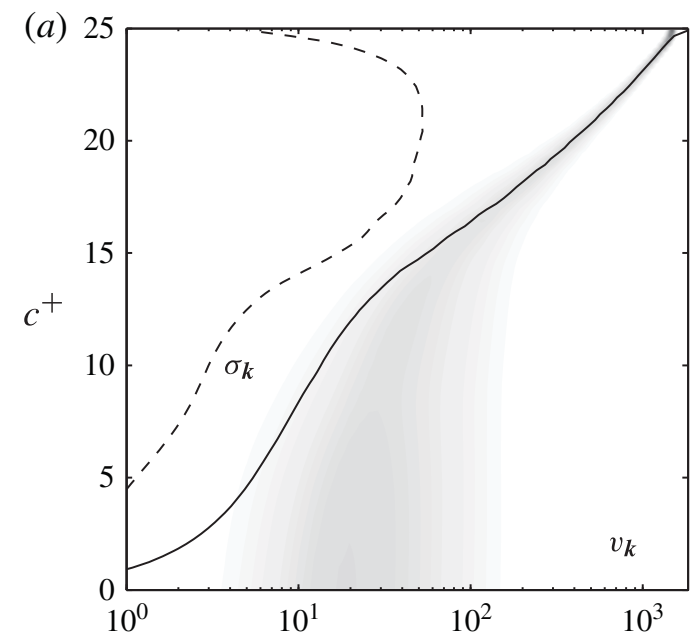

(b)

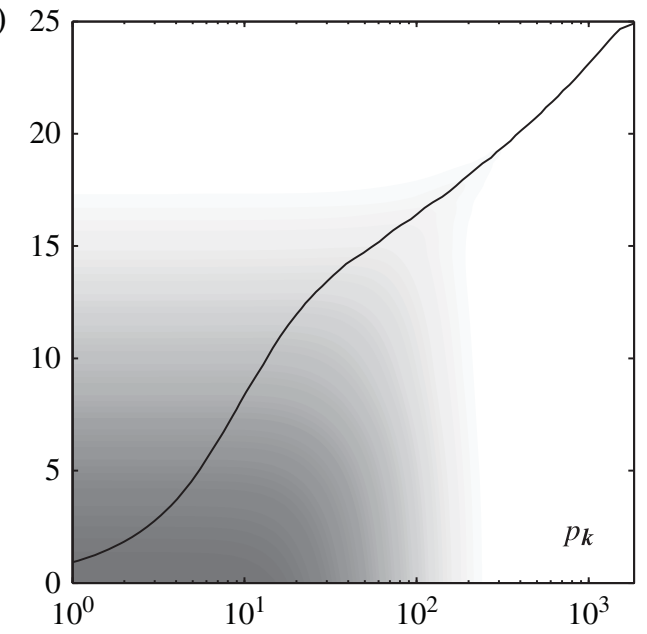

(c)

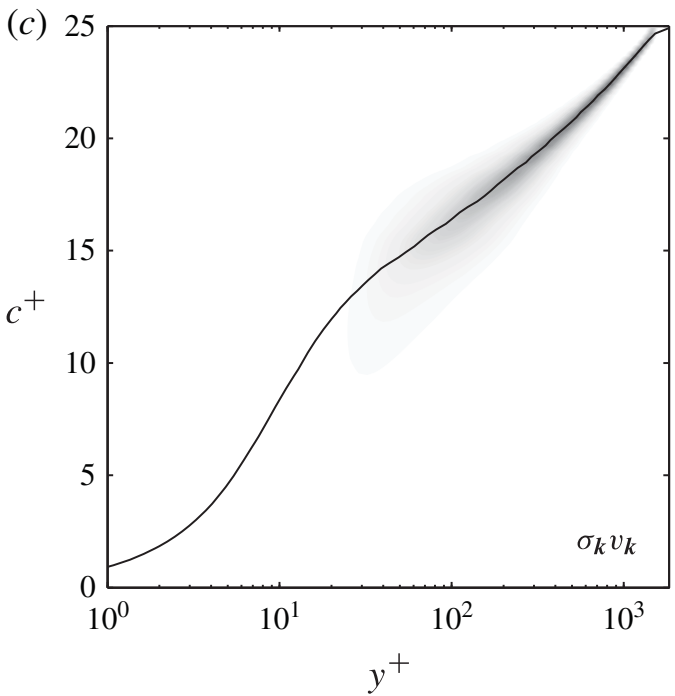

(d)

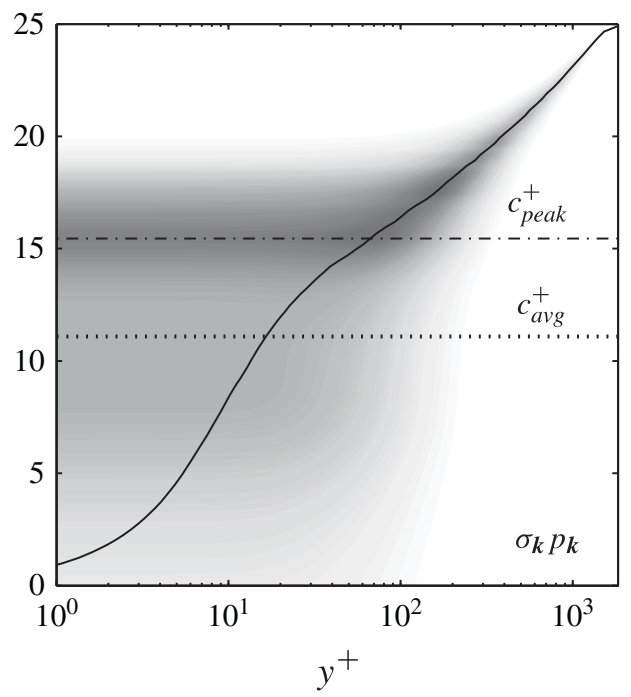

FIGURE 11. Wall-normal distribution of pressure and velocity for modes with $k=n=20$ at $R^{+}=1800\left(\lambda_{x}^{+}=\lambda_{\theta}^{+} \approx 570\right)$. $(a, b)$ The distribution of the normalized velocity $\left(v_{k}\right)$ and pressure field $\left(p_{k}\right)$ for varying speed $c^{+} .(c, d)$ The amplification-weighted velocity $\left(\sigma_{k} v_{k}\right)$ and pressure distributions $\left(\sigma_{k} p_{k}\right)$. In all plots, the solid black line represents the mean velocity profile. The dashed line in $(a)$ shows the variation in singular values with wave speed. Horizontal lines in $(d)$ show the propagation speed corresponding to the peak in wall pressure, $c_{\text {peak }}^{+}$, and a weighted average, $c_{\text {avg }}^{+}$.

for $\lambda_{x}^{+}>400$. For wavelength $\lambda_{x}^{+}=\lambda_{\theta}^{+} \lesssim 400$, velocity structures in the buffer region of the flow have the largest wall-pressure signature, while for wavelength $\lambda_{x}^{+}=\lambda_{\theta}^{+} \gtrsim 400$, velocity structures in the logarithmic region have the largest wall pressure. This abrupt transition can be attributed to the change in behaviour seen from figure 10 to figure 11, i.e. the trade-off between wall proximity and amplification.

Both the peak and average propagation speeds collapse together reasonably well as a function of inner-normalized wavelength for smaller $\left(\lambda_{x}^{+}<400\right)$, slower-moving modes $\left(c^{+}<10\right)$. The peak wall-pressure propagation speeds exhibit some scatter for structures of larger wavelength $\left(\lambda_{x}^{+}=\lambda_{\theta}^{+} \gtrsim 1000\right)$ at higher Reynolds number $\left(R^{+}>\right.$ 2000). However, the average propagation speeds (figure 12b) show smoother trends 

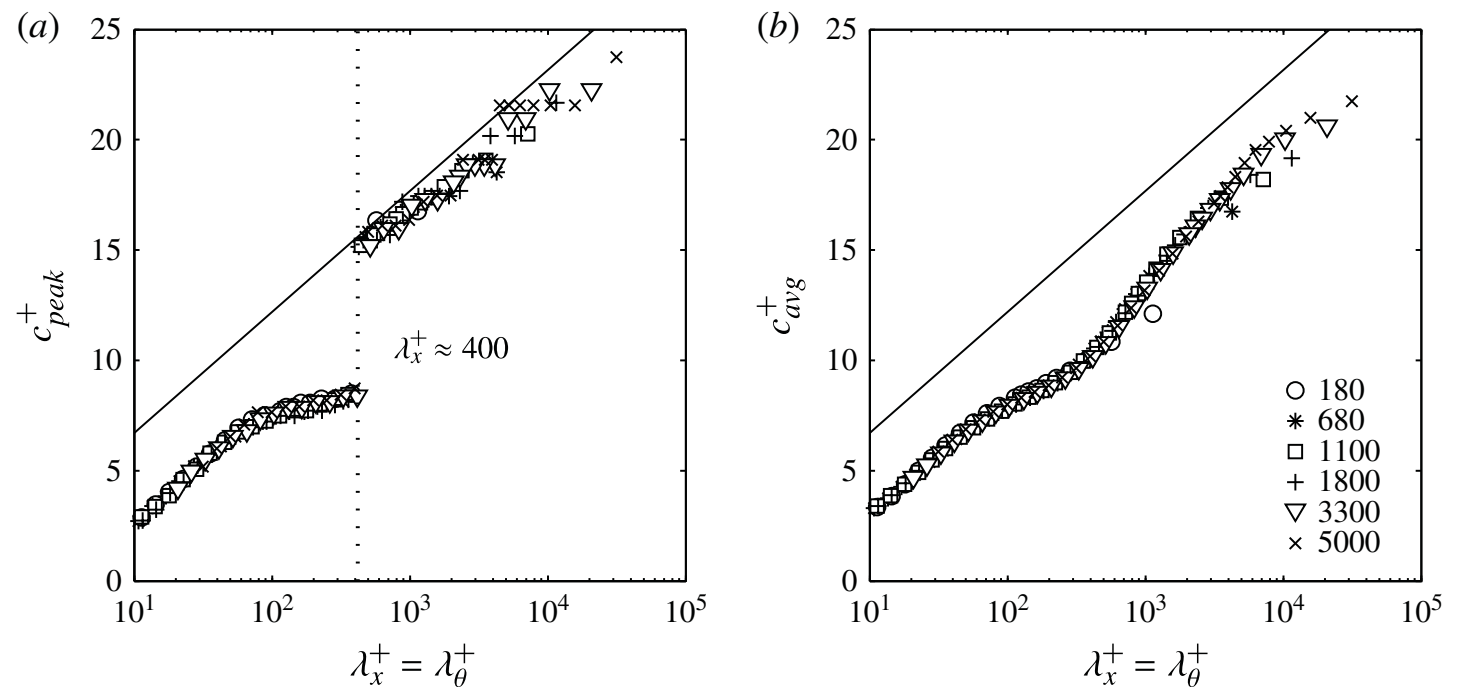

FIGURE 12. Variation in wall-pressure propagation speed for modes with circular aspect ratios $\left(k=n\right.$, or $\left.\lambda_{x}^{+}=\lambda_{\theta}^{+}\right)$for Reynolds number $R e=5000-233000\left(R^{+}=180-5000\right)$, assuming broadband forcing across all wave speeds, $c$. (a) The propagation speed corresponding to the peak in wall pressure, and $(b)$ the weighted average. The solid lines show the prediction based on (4.12) from Panton \& Linebarger (1974), repeated in (5.3).

which suggest that the propagation speeds start to taper off and asymptote to a fixed value at large wavelengths. The inner-normalized wavelengths and propagation speeds at which this transition occurs show a Reynolds-number dependence, indicating that outer or mixed scaling might be more appropriate for these large structures. This scaling will be explored further in future work.

Note that the peak wall-pressure propagation speed for structures with $\lambda_{x}^{+}>400$ agrees reasonably well with the predictions of Panton \& Linebarger (1974) (solid line in figure 12). Specifically, Panton \& Linebarger (1974) proposed that the wall-pressure propagation speed exhibits an overlap region, where:

$$
\begin{aligned}
c^{+} & =-\frac{1}{\kappa} \ln (k \delta)+\frac{1}{\kappa} \ln \left(\frac{u_{\tau} \delta}{v}\right)+B \\
& =\frac{1}{\kappa} \ln \left(\frac{\lambda_{x}^{+}}{2 \pi}\right)+B
\end{aligned}
$$

where $\kappa$ and $B$ are the usual constants in the mean velocity profile (we assume $\kappa=$ 0.42 and $B=5.6$ based on the measurements of McKeon et al. 2004). As shown by the expression on the lower line of (5.3), in essence this assumes that the length scale of the structures that dominate the wall-pressure field scales linearly with $y$ in the logarithmic region (see figure $9 b$ ). The average wall-pressure propagation speeds are lower than the predictions under (5.3). This is likely to be due to the contributions from the slower-moving modes in the near-wall region, where the rank-1 model with broadband forcing is known to be inaccurate. Although we do not expect the rank1 broadband forcing model to yield an accurate quantitative relationship for $c^{+}=$ $f\left(\lambda_{x}^{+}=\lambda_{\theta}^{+}\right)$, it is encouraging to see that it reproduces trends observed in previous studies. Indeed, there is some experimental (Bull 1967) and DNS (Choi \& Moin 1990) support for both the relatively abrupt scale-dependent transition in wall propagation speed (figure 12a), as well as the asymptote towards a near-constant propagation speed for large wall-pressure structures (figure 12b) that scales in outer or mixed units. 


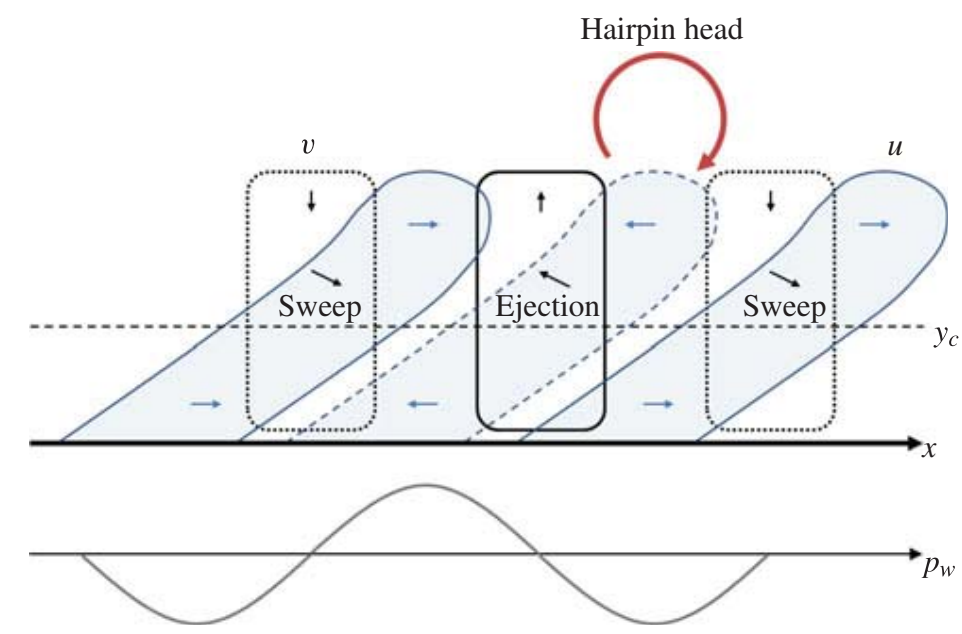

FIGURE 13. Schematic showing the relationship between hairpin-like structures, streamwise velocity (blue), wall-normal velocity (black), and wall pressure (grey) as predicted by the resolvent analysis. See also figures 4,5 and 8.

\section{Conclusion}

The results presented in $\S 3$ show that individual resolvent modes are able to reconcile many of the key relationships among the velocity field, spatial structure, and high-amplitude wall-pressure events observed in previous experiments (Johansson et al. 1987; Ghaemi \& Scarano 2013) and DNS (Kim 1989). These key relationships are summarized schematically in figure 13. This is despite the fact that the resolvent analysis primarily yields the fast component of pressure, arising from the linear interaction between mean shear and wall-normal velocity (as demonstrated by the Green's function solutions, §2.2). However, perhaps the dominance of the fast pressure component points to a stronger result. Although the slow pressure field is known to be energetic throughout the flow (Jimenez \& Hoyas 2008), the forcing-response interpretation of the NSE considered here shows that the slow pressure arises from the non-solenoidal component of the nonlinear forcing term (2.4), (2.16). Under the Helmholtz decomposition, this non-solenoidal forcing does not directly contribute to the velocity field. As a result, correlations between the velocity and pressure fields are expected to be dominated by the fast pressure.

Note that we only consider the velocity and pressure fields associated with rank-1 resolvent modes in this paper. A brief exploration of the pressure fields associated with higher-rank modes (data not shown) indicates that the fast pressure remains dominant for resolvent modes up to at least rank-20, contributing $>90 \%$ of the pressure intensity for all the wavenumber-frequency combinations shown in table 1 . Since the gain-based decomposition does not yield the slow pressure, it appears that the slow pressure must be recovered through closing the loop on the nonlinearity within the resolvent analysis framework, i.e.through an explicit consideration of the triadic interactions that serve to sustain wall turbulence (McKeon et al. 2013).

The resolvent modes also suggest the presence of recurring phase relationships between the velocity and pressure fields across all scales (e.g.figure 3). This has important implications for the wall-based sensing and control of turbulent flows. In particular, most previously proposed control strategies are reliant upon sensing the wall-normal velocity in the bulk of the flow (e.g. opposition control of Choi, Moin \& Kim 1994). Therefore, the fact that the fast wall pressure reflects an integral of the 
wall-normal velocity (2.24) could prove useful in inferring velocity information from wall-based pressure measurements. In addition, recent work by the present authors (Luhar et al. 2013, 2014) suggests that the performance of opposition control can be improved through the inclusion of a phase lag between the sensed wall-normal velocity and the blowing and suction generated at the wall. In this context, the consistent $\pi / 2$ phase difference between the fast pressure and wall-normal velocity may be useful in determining the phase of the wall-based actuation. In general, the fast pressure is likely to be more important than the slow pressure for control purposes due to its direct link to the mean velocity profile and its faster time scale (Sharma et al. 2011). As a result, the fact that the resolvent analysis does not directly yield information regarding the slow pressure may not significantly limit its application to the design and evaluation of effective flow control.

The results pertaining to the three-mode turbulence kernel presented in $\S 4$ show that, compared to the streamwise velocity field, the wall-pressure field is likely to be dominated by structures that are shorter in $x$ and that have near-circular aspect ratios. Further, the turbulence kernel also suggests an apparent amplitude modulation effect in the wall-pressure field, albeit with a different phase relationship between the large and small scales compared to that observed for the streamwise velocity field (Chung \& McKeon 2010). In particular, we expect the small-scale activity in wall pressure to lag the large-scale wall pressure by $\pi / 2$. In other words, we anticipate intense smallscale activity in wall pressure to coincide with regions of increasing large-scale wall pressure (see also Thomas \& Bull 1983). This is in contrast to the observations for the near-wall streamwise velocity, which show that the small-scale activity is most intense in regions of high large-scale velocity (e.g. Marusic et al. 2010). Whether these phase relationships hold in real flows remains to be seen.

Finally, §5 shows that a simple rank-1 model assuming broadband forcing (i.e. velocity and pressure fields approximated by singular value-weighted rank-1 resolvent modes) qualitatively reproduces trends in the wavenumber spectra for streamwise and wall-normal velocity, as well as the pressure field. Consistent with the recent study of Moarref et al. (2013), major differences between the broadband forcing predictions and DNS results (Jimenez \& Hoyas 2008) are concentrated in the near-wall region and close to the pipe centreline. Further, the rank-1 broadband forcing model also provides insight into the scale-dependence of wall-pressure propagation speed (\$5.2), which is important for translating Eulerian measurements into spatial structure. Since there are significant challenges associated with obtaining an accurate characterization of the wall-pressure propagation speeds from experimental measurements at high Reynolds number, low-order models employing resolvent modes could present a viable alternative.

\section{Acknowledgement}

The support of the Air Force Office of Scientific Research under award FA9550-121-0469 (program manager Dr Douglas Smith) is gratefully acknowledged.

\section{REFERENCES}

Ahn, B.-K., GRAham, W. R. \& Rizzi, S. A. 2010 A structure-based model for turbulent-boundarylayer wall pressures. J. Fluid Mech. 650, 443-478.

Del Alamo, J. C. \& Jimenez, J. 2009 Estimation of turbulent convection velocities and corrections of Taylor's approximation. J. Fluid Mech. 640, 5-26. 
Bandhyopadhyay, P. R. \& Hussain, A. K. M. F. 1984 The coupling between scales in shear flows. Phys. Fluids 27 (9), 2221-2228.

Bradshaw, P. \& KoH, Y. M. 1981 A note on Poisson's equation for pressure in a turbulent flow. Phys. Fluids 24, 777.

BuLL, M. K. 1967 Wall-pressure fluctuations associated with subsonic turbulent boundary layer flow. J. Fluid Mech. 28, 719-754.

Chakraborty, P., Balachandar, S. \& Adrian, R. J. 2005 On the relationships between local vortex identification schemes. J. Fluid Mech. 535, 189-214.

Choi, H. \& Moin, P. 1990 On the space-time characteristics of wall-pressure fluctuations. Phys. Fluids 2 (8), 1450-1460.

Choi, H., Moin, P. \& Kim, J. 1994 Active turbulence control for drag reduction in wall-bounded flows. J. Fluid Mech. 262, 75-110.

Chung, D. \& McKeon, B. J. 2010 Large-eddy simulation of large-scale structures in long channel flow. J. Fluid Mech. 661, 341-364.

Dinkelacker, A., Hessel, M., Meier, G. E. A. \& Schewe, G. 1977 Investigation of pressure fluctuations beneath a turbulent boundary layer by means of an optical method. Phys. Fluids 20 (10), S216-S224.

Foias, C., Manley, O., Rosa, R. \& Temam, R. 2001 Navier-Stokes Equations and Turbulence. Cambridge University Press.

GhaEmi, S. \& ScARANO, F. 2013 Turbulent structure of high-amplitude pressure peaks within the turbulent boundary layer. J. Fluid Mech. 735, 381-426.

Guala, M., Metzger, M. \& McKeon, B. J. 2011 Interactions within the turbulent boundary layer at high Reynolds number. J. Fluid Mech. 666, 573-604.

Hu, Z. H., Morfey, C. L. \& SAndham, N. D. 2002 Aeroacoustics of wall-bounded turbulent flows. AIAA J. 40 (3), 465-473.

JACobi, I. \& McKeon, B. J. 2013 Phase relationships between large and small scales in the turbulent boundary layer. Exp. Fluids 54, 1481.

JimeneZ, J. \& Hoyas, S. 2008 Turbulent fluctuations above the buffer layer of wall-bounded flows. J. Fluid Mech. 611, 215-236.

Johansson, A. V., Her, J. Y. \& Haritonidis, J. H. 1987 On the generation of high-amplitude wall-pressure peaks in turbulent boundary-layers and spots. J. Fluid Mech. 175, 119-142.

KIM, J. 1989 On the structure of pressure-fluctuations in simulated turbulent channel flow. J. Fluid Mech. 205, 421-451.

Klewicki, J., Priyadarshana, P. J. A. \& Metzger, M. M. 2008 Statistical structure of the fluctuating wall pressure and its in-plane gradients at high Reynolds number. J. Fluid Mech. 609, $195-220$.

Koumoutsakos, P. 1999 Vorticity flux control for a turbulent channel flow. Phys. Fluids 11, 248.

Luhar, M., Sharma, A. S. \& McKeon, B. J. 2013 A systems approach to modeling opposition control in turbulent pipe flow. In 43rd Fluid Dynamics Conference, American Institute of Aeronautics and Astronautics, June 24-27.

Luhar, M., Sharma, A. S. \& McKeon, B. J. 2014 Opposition control within the resolvent analysis framework. J. Fluid Mech. 749, 597-626.

MARUSIC, I. \& HeUer, W. D. C. 2007 Reynolds number invariance of the structure inclination angle in wall turbulence. Phys. Rev. Lett. 99, 114504.

Marusic, I., Mathis, R. \& Hutchins, N. 2010 Predictive model for wall-bounded turbulent flow. Science 329 (5988), 193-196.

Mathis, R., Hutchins, N. \& MARUsic, I. 2009 Large-scale amplitude modulation of the small-scale structures in turbulent boundary layers. J. Fluid Mech. 628, 311-337.

Mathis, R., Hutchins, N.\& Marusic, I. 2011 A predictive inner-outer model for streamwise turbulence statistics in wall-bounded flows. J. Fluid Mech. 681, 537-566.

McKeOn, B. J., JACOBI, I. \& SHARMA, A. S. 2013 Experimental manipulation of wall turbulence: a systems approach. Phys. Fluids 25, 031301.

McKeon, B. J., Li, J., Jiang, W., Morrison, J. F. \& Smits, A. J. 2004 Further observations on the mean velocity distribution in fully developed pipe flow. J. Fluid Mech. 501, 135-147. 
McKeon, B. J. \& Sharma, A. S. 2010 A critical-layer framework for turbulent pipe flow. J. Fluid Mech. 658, 336-382.

Meseguer, A. \& Trefethen, L. N. 2003 Linearized pipe flow to Reynolds number $10^{7}$. J. Comput. Phys. 186 (1), 178-197.

Moarref, R., Sharma, A. S., Tropp, J. A. \& McKeon, B. J. 2013 Model-based scaling and prediction of the streamwise energy intensity in high-Reynolds-number turbulent channels. J. Fluid Mech. 734, 275-316.

Monty, J. P., Hutchins, N., NG, H. C. H., Marusic, I. \& Chong, M. S. 2009 A comparison of turbulent pipe, channel and boundary layer flows. J. Fluid Mech. 632, 431-442.

O'Farrell, C. \& MARTin, P. 2009 Chasing eddies and their wall signature in DNS data of turbulent boundary layers. J. Turbul. 10, N15.

Panton, R. L. \& Linebarger, J. H. 1974 Wall pressure spectra calculations for equilibrium boundary layers. J. Fluid Mech. 65 (2), 261-287.

Perry, A. E. \& Chong, M. S. 1982 On the mechanism of wall turbulence. J. Fluid Mech. 119, $173-217$.

Perry, A. E., Henbest, S. \& Chong, M. S. 1986 A theoretical and experimental study of wall turbulence. J. Fluid Mech. 165, 163-199.

Perry, A. E. \& MARUSiC, I. 1995 A wall-wake model for the turbulence structure of boundary-layers. 1. Extension of the attached eddy hypothesis. J. Fluid Mech. 298, 361-388.

Schewe, G. 1983 On the structure and resolution of wall-pressure fluctuations associated with turbulent boundary-layer flow. J. Fluid Mech. 134, 311-328.

Sharma, A. S. \& McKeon, B. J. 2013 On coherent structure in wall turbulence. J. Fluid Mech. 728, 196-238.

Sharma, A. S., Morrison, J. F., McKeon, B. J., Limebeer, D. J. N., Koberg, W. H. \& SHERWIN, S. J. 2011 Relaminarisation of $R e_{\tau}=100$ channel flow with globally stabilizing linear feedback control. Phys. Fluids 23 (12), 125105.

SNARSKI, S. R. \& Lueptow, R. M. 1995 Wall pressure and coherent structures in a turbulent boundary layer on a cylinder in axial flow. J. Fluid Mech. 286, 137-171.

Thomas, A. S. W. \& Bull, M. K. 1983 On the role of wall-pressure fluctuations in deterministic motions in the turbulent boundary layer. J. Fluid Mech. 128, 283-322.

Trefethen, L. N. 2000 Spectral Methods in MATLAB. Society for Industrial and Applied Mathematics.

Tsuji, Y., Fransson, J. H. M., Alfredsson, P. H. \& Johansson, A. V. 2007 Pressure statistics and their scaling in high-Reynolds-number turbulent boundary layers. J. Fluid Mech. 585, $1-40$.

Tsuji, Y., Imayama, S., Schlatter, P., Alfredsson, P. H., Johansson, A. V., Marusic, I., Hutchins, N. \& Monty, J. 2012 Pressure fluctuation in high-Reynolds-number turbulent boundary layer: results from experiments and DNS. J. Turbul. 13 (50), 1-19.

WU, X. \& MoIN, P. 2008 A direct numerical simulation study on the mean velocity characteristics in turbulent pipe flow. J. Fluid Mech. 608, 81-112. 